ARTICLE

DOI: $10.1038 / s 41467-018-06309-8$

\title{
LEM4 confers tamoxifen resistance to breast cancer cells by activating cyclin D-CDK4/6-Rb and ER $\alpha$ pathway
}

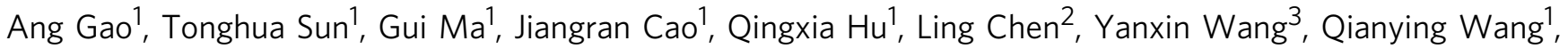

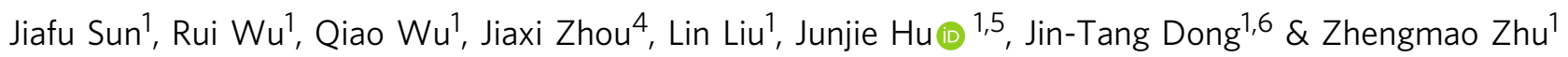

The elucidation of molecular events that confer tamoxifen resistance to estrogen receptor $\alpha$ (ER) positive breast cancer is of major scientific and therapeutic importance. Here, we report that LEM4 overexpression renders ER+ breast cancer cells resistant to tamoxifen by activating the cyclin D-CDK4/6 axis and the ER $\alpha$ signaling. We show that LEM4 overexpression accelerates tumor growth. Interaction with LEM4 stabilizes CDK4 and Rb, promotes Rb phosphorylation and the G1/S phase transition. LEM4 depletion or combined tamoxifen and PD0332991 treatment significantly reverses tamoxifen resistance. Furthermore, LEM4 interacts with and stabilizes both Aurora-A and ER $\alpha$, promotes Aurora-A mediated phosphorylation of $E R \alpha$-Ser167, leading to increase in ER $\alpha$ DNA-binding and transactivation activity. Elevated levels of LEM4 correlates with poorer relapse-free survival in patients with $\mathrm{ER}+$ breast cancer undergoing endocrine therapy. Thus, LEM4 represents a prognostic marker and an attractive target for breast cancer therapeutics. Functional antagonism of LEM4 could overcome tamoxifen resistance.

\footnotetext{
${ }^{1}$ Department of Genetics and Cell Biology, College of Life Sciences, Nankai University, Tianjin 300071, China. ${ }^{2}$ Department of Pathology, Tianjin Central Hospital of Gynecology and Obstetrics, Tianjin 300100, China. ${ }^{3}$ Department of Pharmacology, Yong Loo Lin School of Medicine, National University of Singapore, Singapore, Singapore. ${ }^{4}$ State Key Laboratory of Experimental Hematology, Institute of Hematology \& Blood Diseases Hospital, Chinese Academy of Medical Sciences \& Peking Union Medical College, 288 Nanjing Road, Tianjin 300020, China. ${ }^{5}$ National Laboratory of Biomacromolecules, CAS Center for Excellence in Biomacromolecules, Institute of Biophysics, Chinese Academy of Sciences, Beijing 100101, China. ${ }^{6}$ Department of Hematology and Medical Oncology, School of Medicine, Winship Cancer Institute, Emory University, Atlanta, Georgia. Correspondence and requests for materials should be addressed to J.H. (email: huj@ibp.ac.cn) or to J.-T.D. (email: j.dong@emory.edu) or to Z.Z. (email: zhuzhengmao@nankai.edu.cn)
} 
$\mathrm{T}$ he estrogen receptor (ER) pathway is considered an addictive oncogenic pathway in breast cancer cells. At least $70 \%$ of breast cancers are classified as ER+ breast cancers. Tamoxifen represents a mainstay adjuvant treatment in clinical practice over the past two decades. One-third of breast tumors that initially respond to the adjuvant therapy with tamoxifen will eventually relapse with endocrine-resistant disease ${ }^{1}$. The major mechanisms of endocrine resistance in ER+ breast cancers, through ERa itself, receptor tyrosine kinase (RTK) signaling, or cell cycle regulation with the cyclin $\mathrm{D}-\mathrm{CDK} 4 / 6-\mathrm{Rb}$ pathway, have been demonstrated to be pivotal in endocrine therapy ${ }^{2}$. With regard to the cyclin D-CDK4/6-Rb pathway, the downstream or end points shared by multiple pathways including ERa signaling and RTK signaling could be targeted, which has the benefit of more directly targeting proliferation. The specific CDK4/6 inhibitor PD0332991 combined with endocrine therapy has been shown to substantially improve progression-free survival in patients with ER+ advanced breast cancer $^{3-5}$. Although PD0332991 combined with endocrine therapy was approved as a first-line treatment for advanced ER+ breast cancer by the FDA (2015) and EMA (2016), no reliable biomarkers except ER status has been defined to diagnose tumors that depend on CDK4 activity and respond to CDK4/6 inhibitors ${ }^{6}$.

Cancer cells often exhibit changes in nuclear morphology, and changes in nuclear morphology are a gold standard for clinical cancer diagnosis ${ }^{7}$. Breast cancer cells contain massive nuclear envelope (NE) invaginations ${ }^{8}$. Loss of NE integrity or NE rupturing, which results in genomic instability and uncontrolled exchange of nucleo-cytoplasmic content, may promote cancer progression $^{9-11}$. However, very little is known about the mechanism by which disruption of the NE structure facilitates carcinogenesis and cancer progression. LEM proteins are the better-characterized NE proteins containing the LEM domain that interacts with the highly conserved essential chromatinbinding protein barrier-to-autointegration factor $(\mathrm{BAF})^{12}$. LEM$\mathrm{BAF}$ interactions form an important link between the NE and chromatin to maintain nuclear organization during interphase and in the timing of the post-mitotic NE reformation. LEM2 or LEM4 depletion resulted in nuclear shape defects ${ }^{13,14}$. Moreover, the highly dynamic localization and function of BAF during the cell cycle is tightly regulated by phosphorylation, which is temporally controlled by LEM $4^{13}$. Based on these considerations, we hypothesized that some of the LEM proteins might function as oncoproteins, and any such role could be linked to dysregulation of the cell cycle machinery and activation of cyclin-dependent kinases.

Several studies investigating LEM proteins, including LAP2 and LEM3, have been reported in breast cancer ${ }^{15}$,16. In this study, we present evidence that LEM4 overexpression in ER+ breast cancer cells confers tamoxifen resistance through activation of both the cyclin D-CDK4/6-Rb pathway and the ERa signaling. By studying MCF7-TAMR cells and BT474 cells, we show that elevation of LEM4 expression is a key event to render ER + breast cancer cells resistant to tamoxifen. LEM4 depletion or combined tamoxifen and PD0332991 treatment significantly overcomes the tamoxifen resistance. Moreover, LEM4 interacts with and stabilizes ER $\alpha$, leading to increase in ER $\alpha$ DNA-binding and transactivation activity. Therefore, LEM4 serves as a critical regulator in the transition of ER+ breast cancer cells to estrogen independence and tamoxifen resistance.

\section{Results}

LEM4 predicts clinical outcomes in breast cancer patients. Breast cancer cells often exhibit massive NE invaginations ${ }^{8,17}$. In search of reasons that disruption of the NE structure would benefit a cancer cell, we interrogated the Cancer Genome Atlas database and found that LEM4, a member of the prominent family of NE proteins containing the LEM domain, was significantly overexpressed in breast tumors compared to normal breast epithelium (Fig. 1a, $P<0.001$, Tukey's multiple comparisons test). To investigate the role of LEM4 in breast cancer, we performed immunohistochemistry (IHC) with commercial tissue microarrays (HBre-Duc150-Sur-01/02) and found that LEM4 was more highly expressed in tumor tissues from breast cancer patients and weakly detected in the paired noncancerous tissue regions (Fig. 1b, c). The IHC analysis also demonstrated that, in some cases, LEM4 was highly enriched in the nucleus of tumor cells (Fig. 1b), which is a surprising finding given that LEM4 has been shown to localize to the inner nuclear membrane and endoplasmic reticulum ${ }^{13}$. Moreover, cancer ATLAS analysis with an anti-LEM4 antibody revealed nuclear positivity in some breast cancer cases ${ }^{18}$. Next, we examined the Lem 4 protein by IHC staining of the mouse mammary glands during four different stages (puberty, pregnancy, lactation, and involution). The results showed nuclear negativity in the mouse mammary epithelial cells (Supplementary Fig. 1a). Therefore, translocation occurred in the context of some cancer-related biological events. Although we are unable to reveal the mechanism that led to the nucleoplasm enrichment of LEM4, LEM4 expression significantly increased as tumors progressed to high-grade breast cancer (Fig. 1d). We then investigated whether LEM4 protein expression is associated with overall survival in 284 patients with breast cancer stratified according to breast cancer subtype and ER status. Patients were separated into two groups using the median expression of LEM4 as the dividing line and Kaplan-Meier survival analysis was performed. High LEM4 expression positively correlated with reduced overall survival (Fig. 1e). Patients with high LEM4 expression had greater overall decreased survival rate in luminal B and HER2-enriched breast cancer subtypes (Fig. If, g). In addition, in both ER+ patients and ER- patients, there was a significantly less chance of survival for patients with higher LEM4 expression (Fig. 1h).

We next performed a meta-analysis using an online Kaplan-Meier plotter breast cancer survival analysis to further assess the role of LEM4 in clinical outcomes (www.kmplot.com). We took advantage of the publically gene expression datasets from primary breast cancers with associated clinical data, including disease recurrence and survival (GSE2034 ${ }^{19}$, GSE299020, GSE16446 ${ }^{21}$, and GSE2068522). The results revealed that tumors with higher LEM4 expression had significantly worse relapse-free survival (Fig. 1i). In addition, patients with higher LEM4 expression had greater decreased relapse-free survival in both luminal A and luminal B subtype of breast cancers (Supplementary Fig. 1b). High LEM4 expression also positively correlated with worse overall survival in both ER+ patients and ER- patients (Supplementary Fig. 1c). Thus, increased LEM4 expression significantly correlates with decreased survival of patients with breast cancer.

LEM4 expression promotes breast tumorigenesis. To test the functional relevance of LEM4 overexpression in breast tumors, we stably expressed or depleted LEM4 in breast cancer cells and evaluated the cellular outcomes. Increased LEM4 expression in MCF7 cells (two clones: MCF7-LEM4 \#1 and \#2) enabled the cells to proliferate much faster than control cells (two clones: MCF7-vec \#1 and \#2) in monolayer culture as measured by SRB assay (Fig. 2a). Overexpression of LEM4 in T47D cells also resulted in increased cell growth (Supplementary Fig. 2a). Depletion of LEM4 from MCF7, T47D, BT474 or MCF7-LEM4 cells by RNA interference resulted in significantly decreased cell 
a

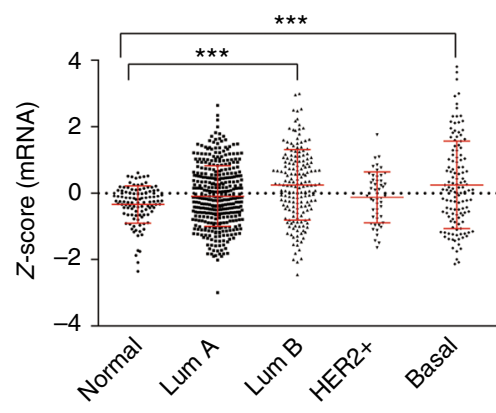

d

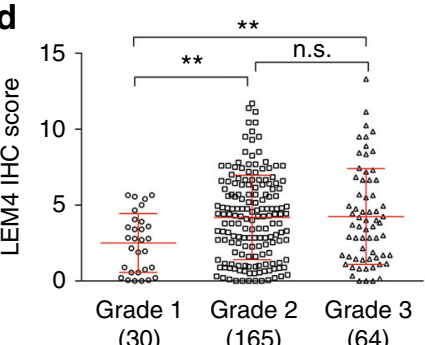

(30) (165) (64)

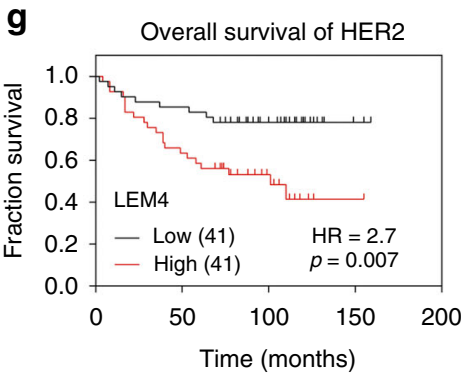

i

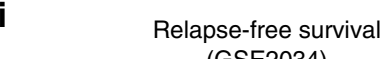
(GSE2034)

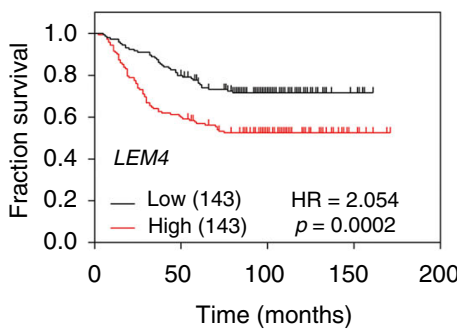

Relapse-free survival (GSE16446)

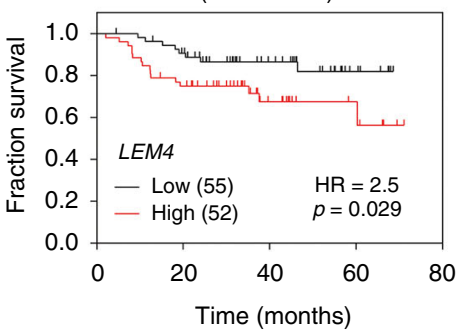

C b

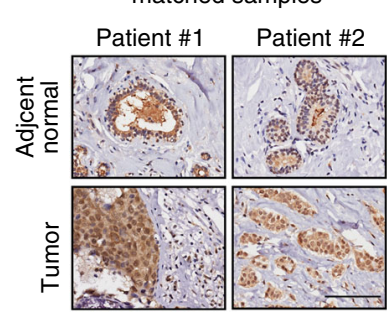

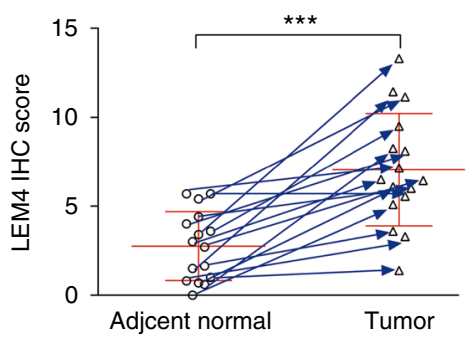

f

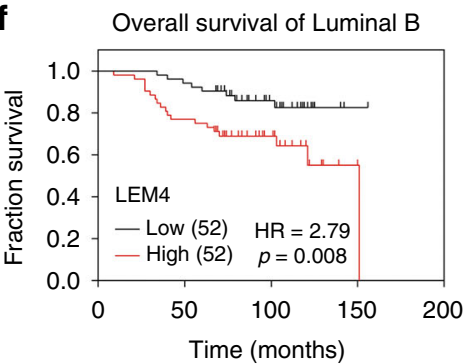

h
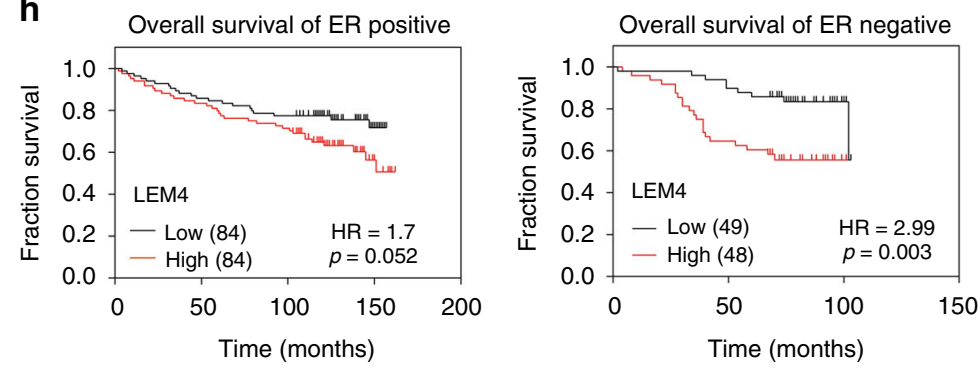
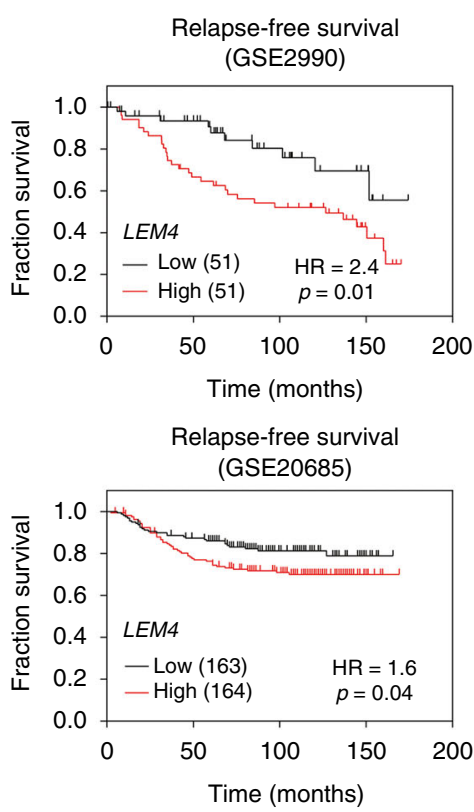

growth (Fig. 2b, c and Supplementary Fig. 2b, c). Similar results were observed in the non-tumorigenic epithelial cells MCF 10A, and RNA interference with LEM4 expression resulted in a significant inhibition of mammosphere formation of MCF 10A cells in matrigel (Supplementary Fig. 2d, e). Evaluation of EdU incorporation showed that elevated LEM4 in MCF7 cells or T47D cells gave rise to an increase in the number of EdU-positive cells (Supplementary Fig. 2f, g). Thus, LEM4 promotes cell proliferation in breast cancer cells and is necessary for cell proliferation in vitro. 
Fig. 1 High LEM4 expression correlates with poor survival of patients with breast tumors. a The Cancer Genome Atlas analysis showed the expression levels of LEM4 across different subtypes of breast cancer and normal tissues. Normal, $n=106$, luminal A, $n=372$, luminal B, $n=174, H E R 2, n=49$, basallike, $n=127$. $\mathbf{b}$ TMA analysis of LEM4 protein expression in breast cancers. Representative IHC images of LEM4 expression in matched normal and primary tumors from two patients are shown. Scale bars, $100 \mu \mathrm{m}$. c Scatter plot showing LEM4 protein expression in 16 paired normal and matched primary tumors, indicated by the blue arrows. d Scatter plots showing the LEM4 immunohistochemical staining results for 259 breast tumors in relation to cancer progression. e-h Kaplan-Meier analysis with median cutoff values of LEM4 expression and overall survival (e) in all 284 patients and patients who had luminal B or HER2 positive subtypes of breast cancer $(\mathbf{f}, \mathbf{g})$ or stratified according to ER $\alpha$ status $(\mathbf{h})$. P-values were calculated by the log-rank test. i Kaplan-Meier analysis with median cutoff values of LEM4 expression for breast cancer from GEO datasets. $P$-values were calculated by the log-rank test. ${ }^{\star \star} P<0.01,{ }^{\star \star \star} P<0.001$, n.s., not significant. Tukey's multiple comparisons test for $\mathbf{a}$, $\mathbf{d}$. Paired $t$-tests for $\mathbf{c}$

Since LEM4 is highly expressed in breast tumors and promotes cell proliferation, we hypothesized that LEM4 might enhance tumorigenesis. To investigate this speculation, we measured the ability of LEM4 to influence colony formation in soft agar. The results showed that MCF7-LEM4 cells had significantly increased colony numbers, whereas LEM4-depleted T47D cells yielded fewer colonies (Fig. 2d, e).

Next, we investigated whether LEM4 accelerates tumorigenesis in vivo with xenografts. MCF7 or T47D-derived cells were injected subcutaneously into athymic nude mice supplemented with a 60-day-release E2 pellet and tumor growth was monitored over time. Compared to MCF7-vec cells, MCF7-LEM4 cells formed faster growing and larger tumors (Fig. 2f). Furthermore, we observed that tumors originating from MCF7-LEM4 cells firmly attached to surrounding tissues with much greater proliferation ability, as indicated by immunostaining with an anti-Ki-67 antibody (Fig. 2h). LEM4-depleted T47D cells formed smaller tumors (Fig. 2g) with significantly lower expression of Ki67 (Fig. 2i). Consistent with these findings, a positive correlation between LEM4 and MKI67 was observed at the mRNA level from the dataset GSE2990 ${ }^{20}(r=0.8544)$ with statistical significance $(P$ $<0.0001$, Pearson's correlation test) (Supplementary Fig. 3). Given that MCF7-LEM4 cells grew as highly invasive tumors firmly attached to surrounding tissues, the MCF7-LEM4 cells were subjected to migration and invasion assays. We observed that MCF7-LEM4 cells were highly invasive in vitro (Supplementary Fig. 4a). Moreover, real-time RT-qPCR analysis showed that Slug and ZEB1, the epithelial-mesenchymal transition makers, were up-regulated in MCF7-LEM4 cells (Supplementary Fig. 4b). Western blot analysis showed that overexpression of LEM4 in MCF7 cells resulted in increased Slug expression (Supplementary Fig. 4c). Furthermore, immunostaining of MCF7-LEM4 cells using antibody (anti E-cadherin) showed the loss of E-cadherin in cell-cell contacts (Supplementary Fig. 4d). Thus, LEM4 overexpression promoted invasive and aggressive growth of MCF7-LEM4 cells.

One of the hallmarks capabilities of cancer is self-sufficiency in growth signals to sustain chronic proliferation ${ }^{23}$. MCF7 cells are estrogen-dependent for growth in vitro and in vivo, and although vector-transfected cells barely survived in estrogen-deprived medium, MCF7-LEM4 cells could grow in steroid-depleted medium (Supplementary Fig. 5a). In vivo, even in the absence of exogenous estrogen supplementation, the MCF7-LEM4 cells generated fast growing tumors with significantly higher expression of Ki-67 in athymic nude mice, whereas MCF7-control cells did not form palpable tumors (Supplementary Fig. 5b, c). Further, a time-course and dosage-course experiment revealed that LEM4 is not an estrogen-responsive gene (Supplementary Fig. 5d). Therefore, LEM4 overexpression enables MCF7 cells to be estrogen-independent for growth.

LEM4 overexpression promotes the G1 to $S$ phase transition. Dysregulated cell division, resulting in aberrant cell proliferation, is one of the key hallmarks of cancer. As LEM4 is a positive regulator of cell proliferation in breast cancer cells, we performed a FACS analysis to address whether LEM4 promotes cell growth and enhances tumorigenesis via alteration of the cell cycle. Cellcycle analysis revealed an increase in the number of cells in G1 phase and a decrease in the number of cells in $S$ phase following depletion of LEM4 in T47D cells (Fig. 3a and Supplementary Fig. 6a). Similar results were observed in BT474 and MCF7 cells (Fig. 3b and Supplementary Fig. 6b, c). However, we observed that the proportion of cells in G1 phase was significantly decreased when LEM4 was overexpressed in MCF7 and T47D cells (Fig. $3 c$ and Supplementary Fig. 6d, e). These data suggest that LEM4 alters the cell cycle by promoting the G1 to S phase transition.

Given that cell cycle progression was altered by modulating expression of LEM4, we sought to determine whether LEM4 regulates the expression of cell cycle-related genes. As LEM4 controls post-mitotic NE formation upon mitotic exit ${ }^{13}$ and accelerates the G1/S phase transition, we focused on the genes for $C D K 1$, cyclin $D, C D K 4 / 6$, cyclin $E$, and $C D K 2$, as well as $R b$ and $E 2 F 1$. Real-time RT-PCR analysis indicated that the CDK1, $C D K 2$, cyclin D1, cyclin E1, Rb, and E2F1 mRNA levels decreased in LEM4-depleted T47D cells (Fig. 3d). In the MCF7-LEM4 cells, the CDK1, CDK2, cyclin D1, cyclin E1, and E2F1 mRNA levels increased significantly (Fig. 3e). Western blot analysis showed that cyclin D1, CDK4, p-CDK4, Rb, and p-Rb decreased in the LEM4 depleted T47D and MCF7 cells (Fig. 3f, Supplementary Fig. 6f). Conversely, the level of cyclin D1, p-CDK4, Rb, p-Rb, E2F1, and cyclin E1 protein expression increased in the MCF7LEM4 and T47D-LEM4 cells (Fig. 3g, Supplementary Fig. 6g). Consistent with these findings, the IHC analysis of tumors showed that cyclin D1, p-CDK4, and p-Rb exhibited a concerted upregulation in the MCF7-LEM4 xenografts and downregulation in T47D-shLEM4 xenografts (Fig. 3h, i). Thus, these data suggest that LEM4 regulates the expression of genes controlling the G1 to $\mathrm{S}$ phase transition.

Overexpression of LEM4 renders cells resistant to tamoxifen. The gene expression signatures representing cell cycle progression can predict disease outcome in women treated with tamoxifen and suggests a possible mechanism for endocrine resistance ${ }^{24}$ Given that LEM4 overexpression enabled MCF7 cells to be estrogen-independent for growth, and the expression of CDK1, cyclin D1, CDK4/6, and CDK2, cyclin E were up-regulated in both MCF7-LEM4 and T47D-LEM4 cells, we sought to determine whether the LEM4 overexpression could account for tamoxifen resistance in ER + breast cancers. We found elevated levels of LEM4 protein in MCF7-TAMR cells as compared to MCF7 cells (Fig. 4a). We then examined LEM4 mRNA levels in the dataset GSE $100075^{25}$ from LTED models. The results revealed that LEM4 expression was significantly elevated in MCF7-LTED models (Fig. 4b). In agreement with previous reports, tamoxifen alone had minimal effect on cell proliferation in MCF7-TAMR cells. However, siRNA knockdown of LEM4 was sufficient to inhibit cell proliferation with enhanced sensitivity to tamoxifen (Fig. 4c). 
a
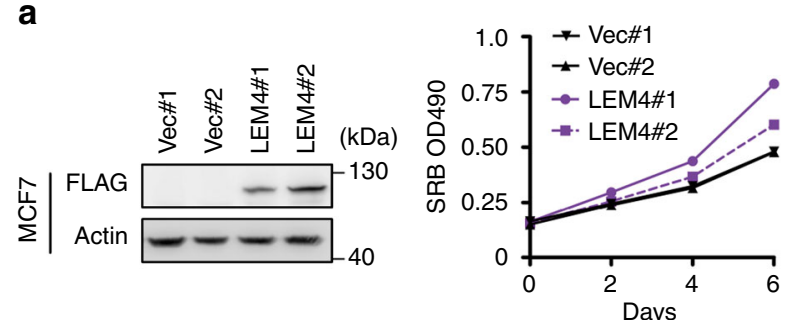

C
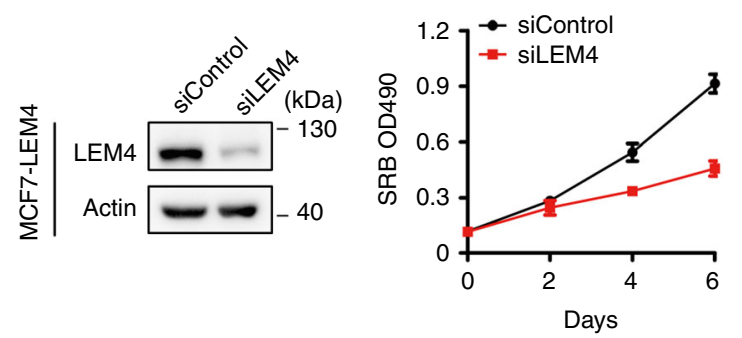

b

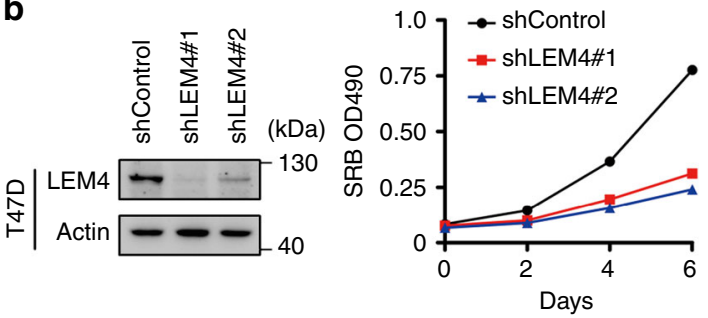

d

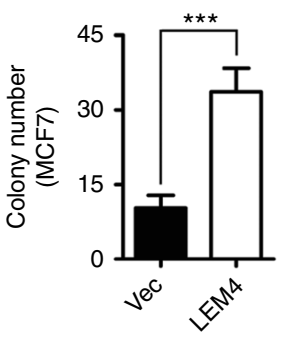

e

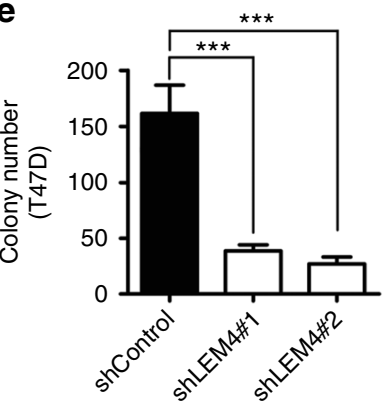

f
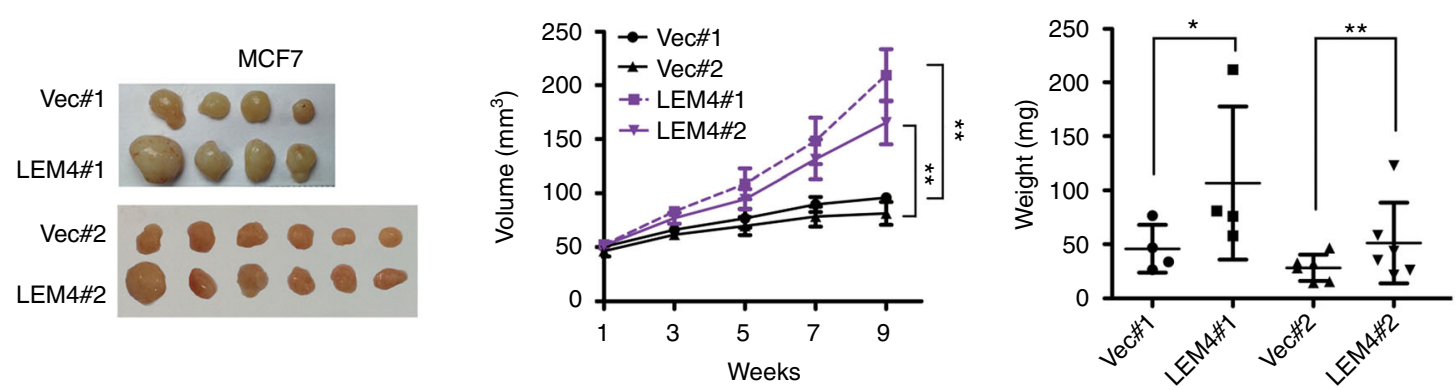

g
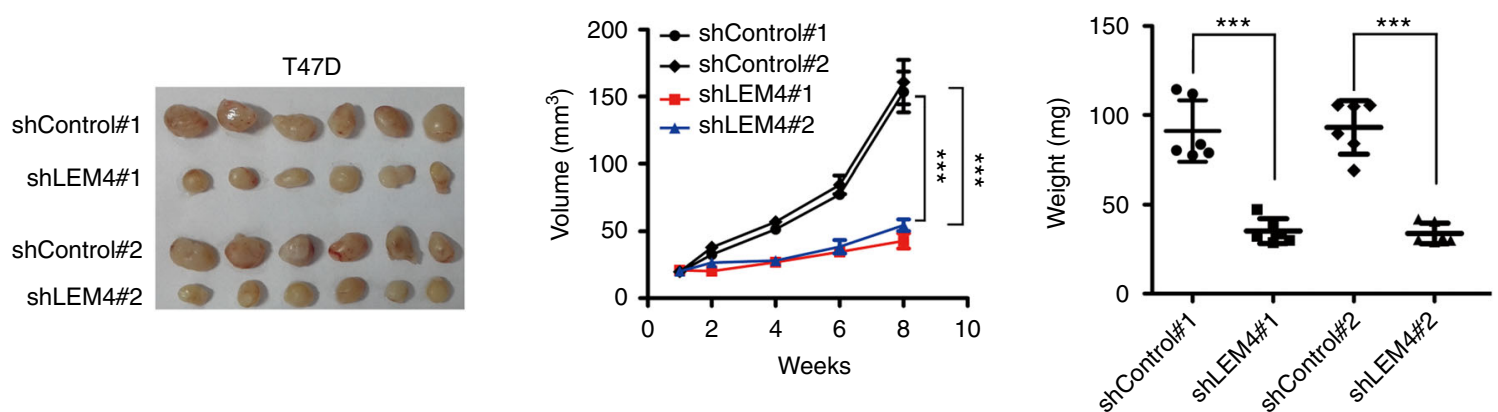

h

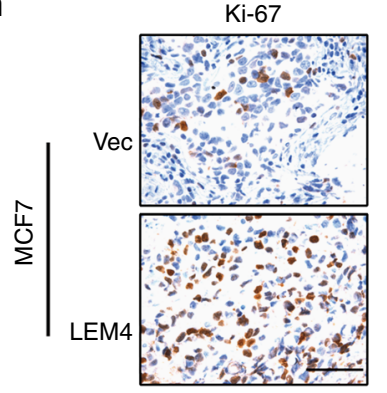

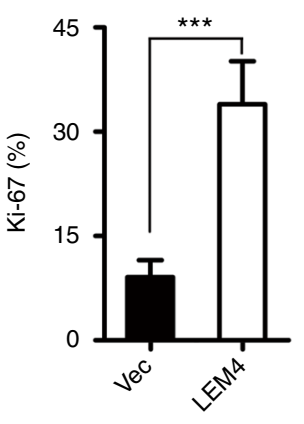

i

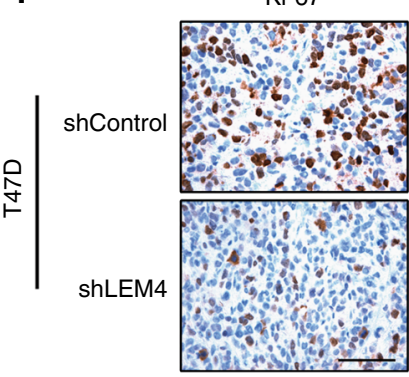


Fig. 2 LEM4 promotes cell proliferation and tumorigenesis. a Growth curve of MCF7-vec and MCF7-LEM4 cells were measured by SRB assay in monolayer culture. Immunoblot was performed with anti-LEM4 antibody. b Growth curves of shControl-T47D, shLEM4\#1-T47D, and shLEM4\#2-T47D cells were measured by SRB assay in monolayer culture. Immunoblot was performed with anti-LEM4 antibody. c MCF7-LEM4 cells were transfected with LEM4 siRNA and control siRNA. Western blot was performed with anti-LEM4 antibody. Growth curves were measured by SRB assay in monolayer culture. d, e Soft agar colony formation by MCF7-vec and MCF7-LEM4 cells (d) or by T47D-shcontrol and T47D-shLEM4 cells (e). $\mathbf{f}, \mathbf{g}$ Tumor growth of MCF7 and T47D cells implanted subcutaneously in athymic mice in the presence of an exogenous slow release estrogen implant. Mean \pm s.e.m, $n=4$ or 6 for MCF7 cells (f), $n=6$ for T47D cells ( $(\mathbf{g})$. h, i IHC for ki-67 in subcutaneous xenograft tumors from Figs. 2f, g. Mean \pm s.d. for three independent replicates. Scale bars, $50 \mu \mathrm{m} .{ }^{\star} P<0.05,{ }^{\star \star} P<0.01$, and ${ }^{\star \star \star} P<0.001$. Repeated measures ANOVA for $\mathbf{f}$ (volume), $\mathbf{g}$ (volume). Student's t-test for $\mathbf{f}$ (weight), $\mathbf{g}$ (weight), $\mathbf{h}, \mathbf{i}$

independent ERa activity. Given depletion of LEM4 in BT474 cells inhibited cell proliferation, we then investigated whether LEM4 overexpression is a key event in tamoxifen resistance through HER2 expression. We depleted LEM4 expression with LEM4 siRNA in BT474 cells. The results revealed that LEM4 depletion did not alter the expression of HER2 (Supplementary Fig. 7a). Conversely, the LEM4 levels decreased upon knockdown of HER2 with HER2 siRNA in BT474 cells (Supplementary Fig. 7a). Reduction of HER2 expression in BT474 cells by siRNAs enhanced sensitivity to tamoxifen (Supplementary Fig. 7b). In consistence with this finding, treatment of LEM4-depleted BT474 cells with tamoxifen resulted in significant cell death with IC50 values from greater than $4 \mu \mathrm{mol} \mathrm{L}^{-1}$ to $120 \mathrm{nmol} \mathrm{L}^{-1}$ (Fig. $4 \mathrm{e}$ ). Thus, knockdown of LEM4 enhances tamoxifen anti-tumor effects in both MCF-TAMR and BT474 cells.

We next determined whether overexpression of LEM4 sufficed to induce tamoxifen resistance in vivo. Notably, unlike MCF7 cells, growth of MCF7-LEM4 cells as xenografts in immunodeficient mice failed to respond to the cytostatic/cytotoxic inhibition effects of tamoxifen (Fig. 4f). However, xenografts of BT474shLEM4 cells regained sensitivity to tamoxifen and exhibited significant tumor regression (Fig. 4g). As LEM4 overexpression enabled MCF7 cells to be tamoxifen resistant, we investigated whether LEM4 overexpression in primary breast tumors may prognosticate subsequent tamoxifen resistance. We analyzed the GEO datasets (GSE299020, GSE349426, and GSE919527) of which the patients treated with adjuvant tamoxifen monotherapy (exclude all chemotherapy). We defined each dataset into two groups with respectively high and low level of LEM4. The Kaplan-Meier survival analysis results revealed that the group expressing high levels of LEM4 displayed a higher probability to develop recurrence as compared to the low group (Fig. 4h). Therefore, these data indicate that overexpression of LEM4 confers tamoxifen resistance.

LEM4 activates the cyclin D-CDK4/6-Rb axis. In culture, tamoxifen treatment leads to a G1 phase-specific cell cycle arrest and a consequence reduction in cell proliferation ${ }^{28}$. The actions of CDK4/6, through the phosphorylation of $\mathrm{Rb}$, are pivotal in the transition from G1 to $\mathrm{S}$ phase in $\mathrm{ER}+$ breast cancer cells ${ }^{29}$. Overexpression of LEM4 in MCF7 cells alters the phosphorylation of both CDK4 and Rb. In addition, analysis of BT474 cells as subcutaneous tumors treated with shRNA targeting LEM4 plus tamoxifen for 6 weeks showed significantly decreased expression of p-CDK4 and p-Rb (Supplementary Fig. 8a). To investigate whether PD0332991 was able to overcome the tamoxifen resistance induced by LEM4 overexpression, we treated MCF7-LEM4 cells and MCF7-TAMR cells with tamoxifen and PD0332991 alone or in combination and monitored cell survival. Combination treatment of cells resulted in significantly reduced cell growth in MCF7-LEM4 and MCF7-TAMR cells under estrogen-depleted conditions, as well as decreased p-Rb levels (Fig. 5a, b). Similar results were observed in BT474 cells (Supplementary Fig. 8b).
Next, we determined whether PD0332991 overcomes the tamoxifen resistance of MCF7-LEM4 cells in vivo. Tumor xenografts were established by injecting MCF7-LEM4 cells subcutaneously into athymic nude mice with estrogen supplementation. The mice were randomized to tamoxifen treatment, PD0332991 treatment or combined treatment until tumors reached an approximate volume of $100 \mathrm{~mm}^{3}$. The growth of MCF7-LEM4 cells remained unaffected by tamoxifen treatment alone as in the xenografts, but was suppressed by PD0332991, and the drug combination induced near-complete tumor regression (Fig. 5c). Analysis of tumors treated with PD0332991 plus tamoxifen for 6 weeks revealed reduced tumor cell density and increased fibrosis (Fig. 5d, H\&E). Tumors treated with PD0332991 or the combination exhibited a decrease in Ki67+ tumor cells compared to the tamoxifen-treated tumors. Moreover, PD0332991 induced apoptosis (IHC analysis showed an increase in cleaved caspase-3/7-positive tumor cells with combined tamoxifen and PD0332991 treatment). p-CDK4 and p$\mathrm{Rb}$ levels decreased similarly as in the xenografts of LEM4depleted cells (Fig. 5d).

Given LEM4 is not a transcription factor and the role of LEM4 in the complex regulatory network modulating $\mathrm{p}-\mathrm{Rb}$ function is unclear. We initially performed GST-pull down assays to test whether LEM4 binds to CDK4 and Rb. The results showed that GST-LEM4, but not GST, could pull-down CDK4 and Rb (Fig. 5e). We also performed co-immunoprecipitation (Co-IP) experiments in HEK293T cells following transfection of FLAGCDK4 and GFP-LEM4 and found that GFP-LEM4 interacted with FLAG-CDK4 (Fig. 5f). In MCF7 cells, endogenous Rb was readily detected in FLAG-LEM4 immunoprecipitates (Fig. 5g). These data indicate that LEM4 binds to CDK4 and Rb. We further investigated whether loss of LEM4 results in CDK4 and $\mathrm{Rb}$ instability. We measured the half-life of CDK4 and Rb using a cycloheximide (CHX) chase assay. Degradation of both $\mathrm{Rb}$ and CDK4 was significantly aggravated at each time point in the LEM4-depleted cells (Fig. 5h). Overall, these data show that LEM4 enhances the stability and phosphorylation of $\mathrm{Rb}$ to promote the transition from $\mathrm{G} 1$ to $\mathrm{S}$ phase, resulting in tamoxifen resistance (Fig. 5i).

LEM4 induces ERa transactivation activity. Cyclin D1 and c-Myc were significantly upregulated in both MCF7-TAMR and MCF7-LEM4 cells (Fig. 6a). Remarkably, LEM4 knockdown reduced the expression of both cyclin D1 and c-Myc significantly in the two cell models (Fig. 6b). Cyclin D1 has been established as a major target of ERa, and cyclin D1 overexpression is associated with tamoxifen resistance ${ }^{30,31}$. To investigate whether LEM4 enhances ERa transactivation, MCF7 cells were transfected with LEM4 and ERE-luc, or ERa-negative MDA-MB-231 cells were transfected with LEM4, ERa, and ERE-luc. The reporter assay revealed LEM4 significantly enhanced ERa transactivation activity (Fig. 6c). To further explored the role of LEM4 in regulating the interaction of ER $\alpha$ with chromatin at the promoters of 

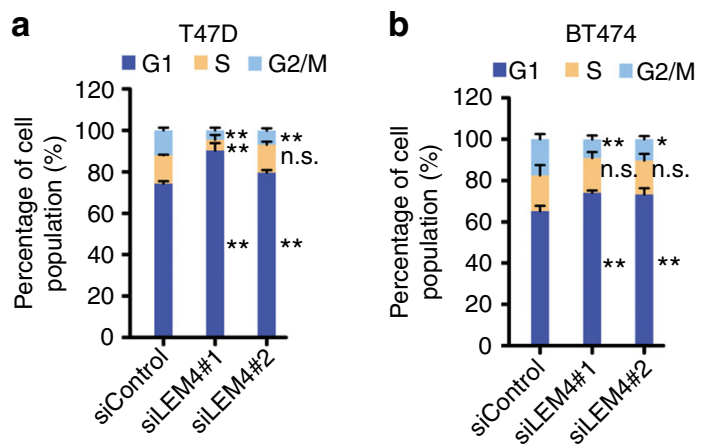

d

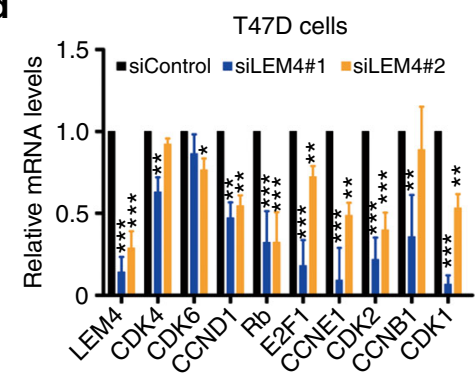

e

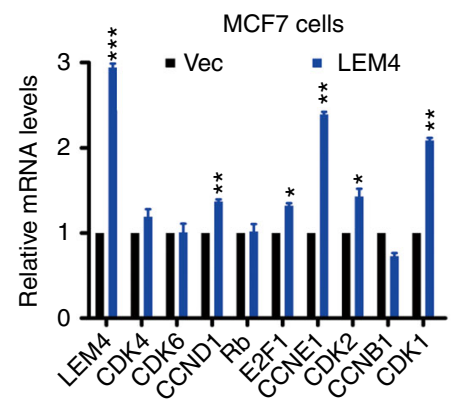

h

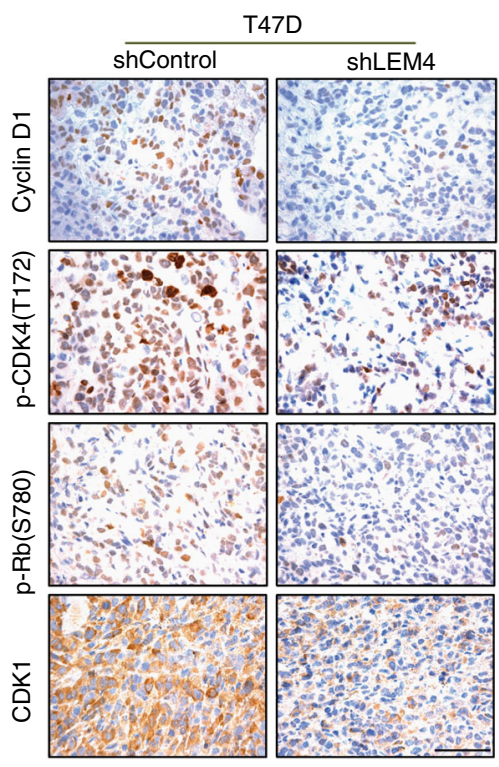

$f$

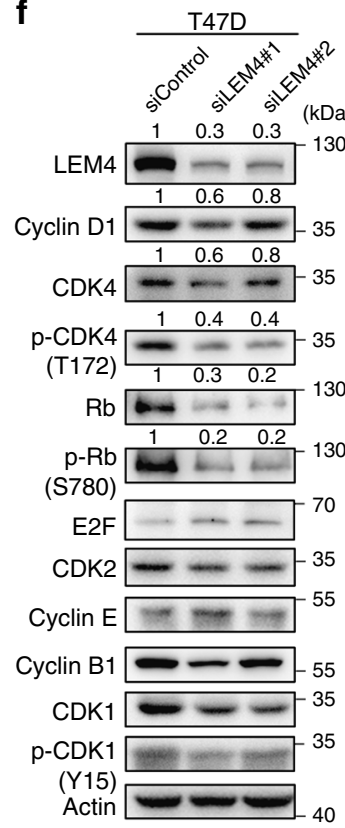

MCF7

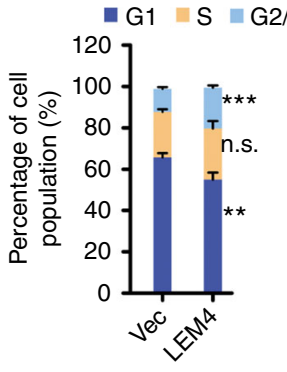


a

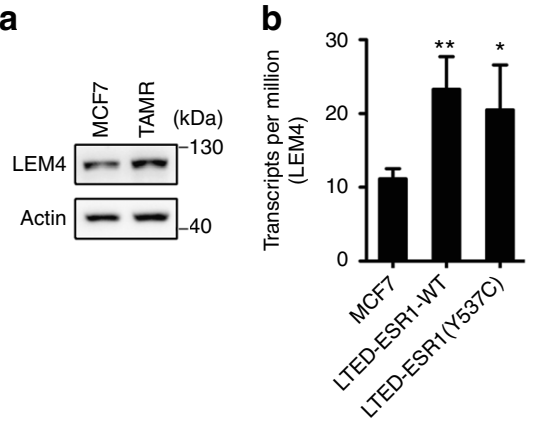

c

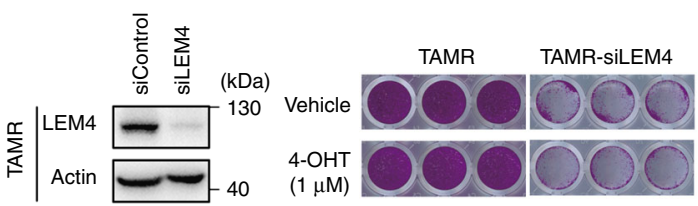

d

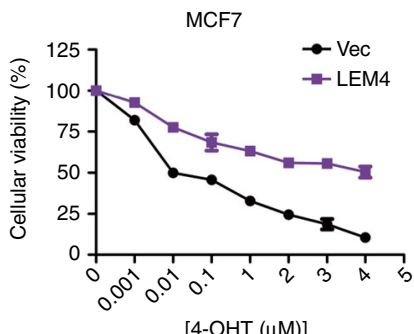

f

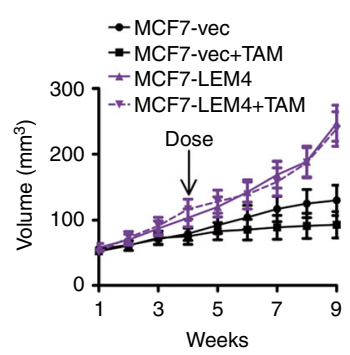

g

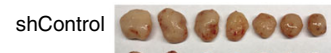

shLEM4 000000

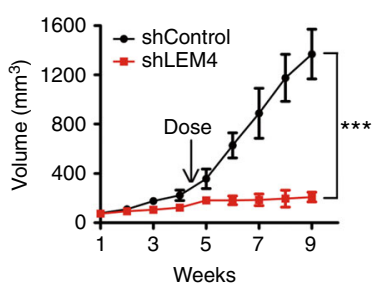

h

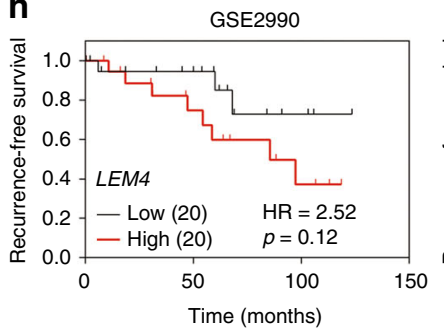

e

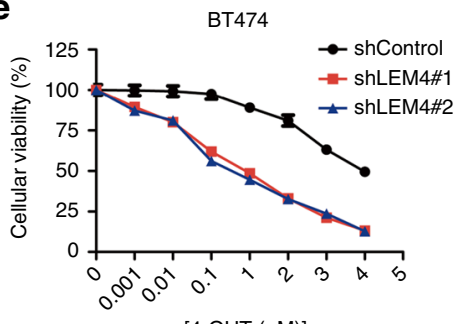

[4-OHT $(\mu \mathrm{M})]$
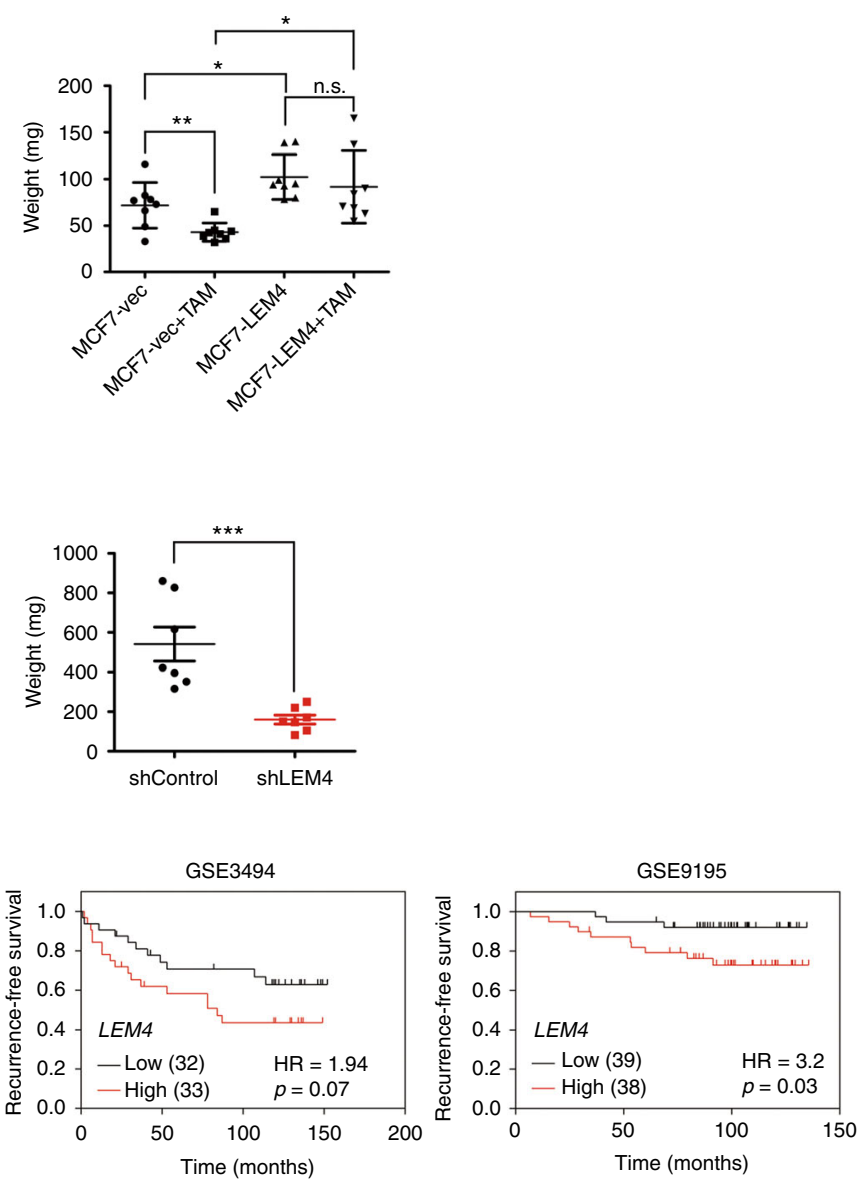

ER $\alpha$ target genes, we performed ER $\alpha$ chromatin immunoprecipitation (ChIP) analysis of known ERa-binding regions in the ERa target genes loci, TFF1, PR, GREB1, CCND1, and $c-M y c$. Following estrogen treatment for $45 \mathrm{~min}$, the occupancy of ERa to the ERa-binding sites was significantly enhanced in MCF7LEM4 cells (Fig. 6d). To gain more insight into the role of LEM4 on enhancing the recruitment of ERa at the promoters of ER target genes, a time course ChIP analysis was performed to

compare the kinetics of estrogen-stimulated loading of endogenous ER $\alpha$ at the promoter of ER $\alpha$ target genes. In MCF7 cells, ERa was recruited to the promoter of $P R$ and GREB1 in a dynamic fashion. In detail, ERa became rapidly bound to the promoter of $P R$ and GREB1, within $15 \mathrm{~min}$ following E2 stimulation, the binding peaked by $30 \mathrm{~min}$ and had declined gradient by $60 \mathrm{~min}$. While in MCF7-LEM4 cells, we observed a significant amount of ERa was present at the promoter of ER 
Fig. 4 LEM4 promotes tamoxifen resistance in ER positive breast cancers. a Western blot was performed with LEM4 antibody in MCF7 and MCF7-TAMR cells. b The GEO GSE100075 was downloaded from Gene Expression Omnibus (GEO). The expression level of LEM4 in MCF7, MCF7-LTED-ESR1(WT), and MCF7-LTED-ESR1(Y537C) cells was measured by transcription per million (TPM). c LEM4 siRNA and control siRNA treated MCF7-TAMR cells were treated with or without tamoxifen $(1.0 \mu \mathrm{M})$ for 5 days. Total cell viability were assessed by SRB assays. Experiments were repeated three times, each experiment was triplicates. $\mathbf{d}$ A cellular viability assay was performed in MCF7-LEM4 and MCF7-vec cells treated with various concentrations of 4-OHT for 7 days. e BT474-shcontrol and BT474-shLEM4 cells were treated with various concentrations of 4-OHT for 7 days. Cellular viability was assessed by SRB assays. $\mathbf{f}$ Tumor growth and tumor weight of MCF7-vec and MCF7-LEM4 cells as subcutaneous xenografts in athymic mice with E2 supplementation until tumors reached $100 \mathrm{~mm}^{3}$, then treated with or without tamoxifen (TAM) pellets implanted subcutaneously for 30 days. Mean \pm s.e.m., $n=8$. $\mathbf{g}$ Tumor growth of BT474-shControl and BT474-shLEM4 cells as subcutaneous xenografts in athymic mice with E2 supplementation until tumors reached $\sim 200$ $\mathrm{mm}^{3}$, then treated with tamoxifen pellets implanted subcutaneously. Mean \pm s.e.m., $n=7$. h Three breast cancer datasets (GSE2990, GSE3494 and GSE9195) were from the KM Plotter database (www.kmplot.com). Kaplan-Meier analysis of recurrence-free survival in the cohorts of patients treated with adjuvant tamoxifen monotherapy (exclude all chemotherapy). Samples were stratified into "high" and "low" LEM4 expression based on median cutoff value in each dataset. $P$-values were calculated by the log-rank (Mantel-Cox) test. n.s., not significant, ${ }^{\star} P<0.05,{ }^{\star \star} P<0.01$ Tukey's multiple comparisons test for b, $\mathbf{f}$ (weight). Repeated measures ANOVA for $\mathbf{g}$ (volume), Student's $t$-test for $\mathbf{g}$ (weight)

target genes, and the signal of ERa at the promoter was relatively constant until later time points. Strikingly, a less amount of the signal of ERa (at the promoter of CCND1 and GREB1 gene) or no signal of ERa (at the promoter of $P R$ gene) was detected at the same locus until late time points in the LEM4-depleted MCF7LEM4 cells (Fig. 6e). Therefore, these data suggest that LEM4 activates ER $a$ transactivation activity and that LEM4-induced ER $\alpha$ activation could not be inhibited by tamoxifen.

LEM4 interacts with and stabilizes ERa. The mechanisms by which LEM4-induced ER $\alpha$ transactivation activity is an interesting question. We observed a higher level of ERa and p-ERaSer167 in both MCF7-LEM4 and MCF7-TAMR cells compared to the control MCF7 cells (Fig. 6a). Conversely, LEM4 knockdown reduced both ERa and p-ERa-Ser167 levels (Fig. 6b). Similar results were observed in LEM4-depleted BT474 cells (Supplementary Fig. 9a). In agreement with these findings, we observed an elevated abundance of p-ERa-Ser167 in MCF7LEM4 xenografts and decreased abundance in BT474-shLEM4 xenografts (Supplementary Fig. 9b). Understanding that human ERa is rapidly degraded in mammalian cells in an estradioldependent manner ${ }^{32}$. We investigated whether LEM4 could prevent ER $\alpha$ degradation. MCF7-LEM4 cells were treated with E2 for $30 \mathrm{~min}$. Interestingly, we observed that $\mathrm{ER} \alpha$ was not degraded in MCF7-LEM4 cells (Fig. 7a). To evaluate whether LEM4 reduction is related to ERa stability, we measured the half-life of ERa using a CHX chase assay. As shown in Fig. 7b, degradation of ERa was accelerated at each time point in LEM4-depleted cells. These data suggested that LEM4 might interact with ERa to prevent ERa degradation. As shown in Fig. 7c, LEM4 was detected in ERa immunoprecipitates in BT474 cells. Furthermore, Co-IP and GST pull-down assays revealed a direct interaction between LEM4 and ER $\alpha$, and the interaction between ER $\alpha$ and LEM4 occurred at the DNA-binding domain conclude Serine-167 (Fig. 7d, e). Moreover, immunofluorescence staining for ERa and LEM4 in MCF7-LEM4 (FLAG tagged) cells showed that ERa colocalized with LEM4 not only in the NE but also in cytoplasm (Fig. 7f). Thus, LEM4 physically interacts with and stabilizes ERa.

In addition, we determined whether unliganded ERa is required for the estrogen-independent growth of MCF7-LEM4 cells. MCF7-LEM4 cells were transfected with siRNA of ESR1 or treated with fulvestrant for 6 days, we found that downregulation of ER $\alpha$ inhibited estrogen-independent growth of MCF7-LEM4 cells (Fig. 7g). A further exploration in dataset GSE33658, which was designed for a phase II neoadjuvant trial of anastrozole (A), fulvestrant $(\mathrm{F})$ and gefitinib $(\mathrm{G})$ in patients with newly diagnosed ER+ breast cancer ${ }^{33}$. As shown in Fig. $7 \mathrm{~h}$, at the post-treatment in both AF-treatment and AFG-treatment group, LEM4 mRNA level reduced in the patients with complete response or the partial response disease-state, while the expression level of LEM4 increased in the patient with progressive disease-state.

LEM4 mediates the phosphorylation of ERa-Ser167 by AuroraA. Phosphorylation of ERa-Ser167 has been shown to sufficiently upregulate cyclin $\mathrm{D} 1^{34-36}$. Given the phosphorylation level of ERa-Ser167 was significantly altered when LEM4 was overexpressed or depleted in ER+ breast cancer cells, we then determined whether LEM4 could directly regulate the phosphorylation of ER $\alpha-S e r 167$. ER $\alpha-S e r 167$ has been shown to be phosphorylated by Aurora-A, AKT, and S6K1 ${ }^{34-36}$. We observed that only Aurora-A increased in the MCF7-LEM4 cells, and a large amount of p-Aurora-A was induced in MCF7-LEM4 and MCF7-TAMR cells (Fig. 8a). Whereas both Aurora-A and p-Aurora-A decreased significantly in LEM4-depleted MCF7TAMR cells or the LEM4-aboragated MCF7-LEM4 cells (Fig. 8b). Similar results were observed in BT474-shLEM4 cells (Fig. 8c). Next, we assessed the levels of p-ERa-Ser167 in MCF7-LEM4 cells treated with siRNAs against AKT, Aurora-A, and LEM4 respectively. Immunoblot analysis showed that LEM4 or AuroraA depletion resulted in a great reduction in p-ERa-Ser167 (Fig. 8d). Given that Aurora-A interacts with and phosphorylates $\mathrm{ERa}^{36}$, we determined whether LEM4 actively interacts with Aurora-A to phosphorylate ERa. Co-IP revealed that LEM4 interacts with Aurora-A (Fig. 8e). Furthermore, we performed Co-IP in LEM4-depleted HEK293T cells following the transfection of FLAG-ER $\alpha$ and GFP-Aurora-A. The results demonstrated that depletion of LEM4 decreased the interaction between ERa and Aurora-A in vivo (Fig. 8f). Moreover, the CHX chase assay revealed that LEM4 contributes to the stability of the Aurora-A protein in both MCF7 cells and BT474 cells (Fig. 8g). Thus, LEM4 enhances Aurora-A-mediated phosphorylation of ERa on Ser167 and promotes ERa mediated transcription of CCND1 and $c-M y c$ (Fig. 8h).

\section{Discussion}

Endocrine therapy is the cornerstone of treatment for patients with $\mathrm{ER}+$ breast cancer ${ }^{37,38}$. However, the emergence of resistance to long-term endocrine treatment is inevitable in a proportion of patients with advanced breast cancer. The major challenge for successful treatment remains to identify new therapeutic targets or more specific biomarkers that are predictive of the therapeutic responses to endocrine therapy. Here, we characterize a critical role of LEM4 overexpression in tamoxifen resistance. Firstly, LEM4 accelerates malignant cell growth and breast tumorigenesis. Moreover, the overexpression of LEM4 enables MCF7 cells to be estrogen-independent for growth. 

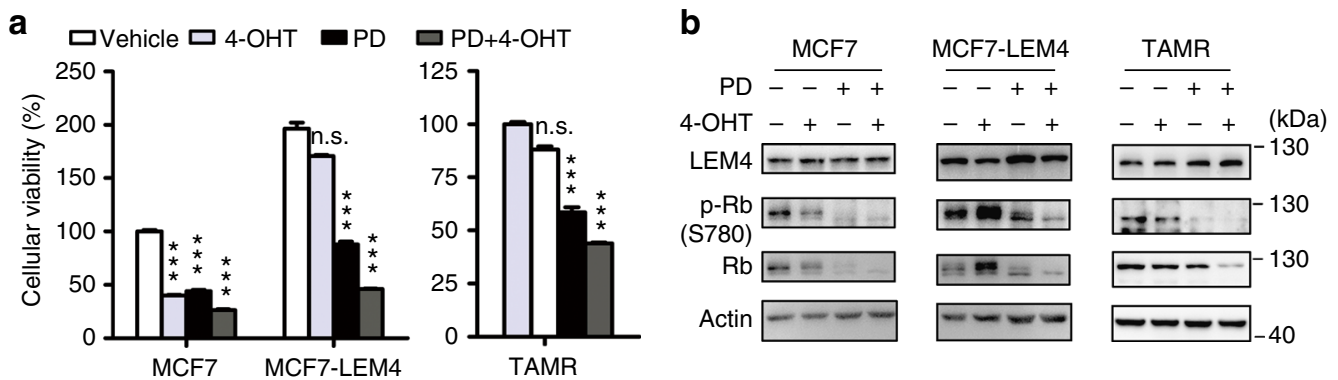

C
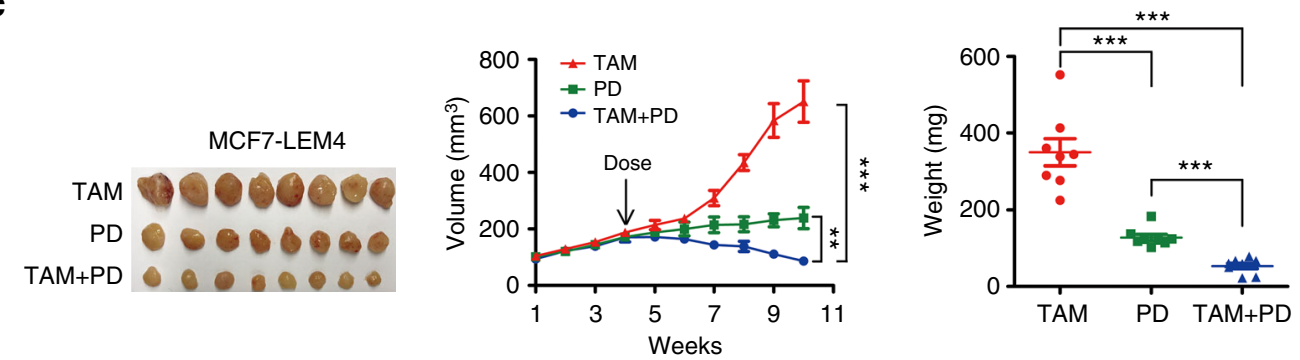

d

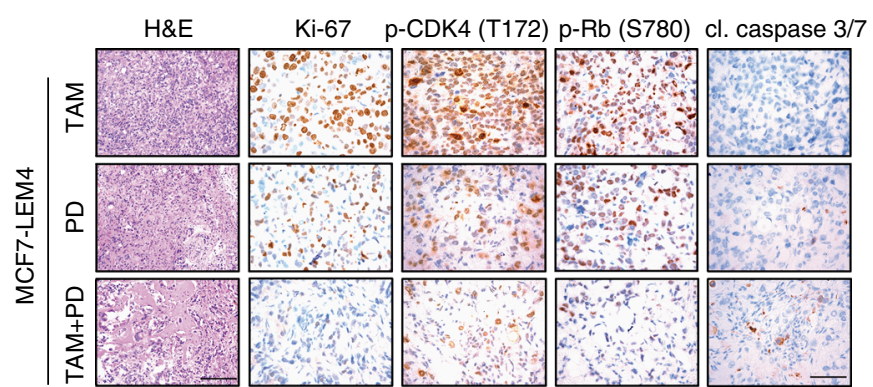

e

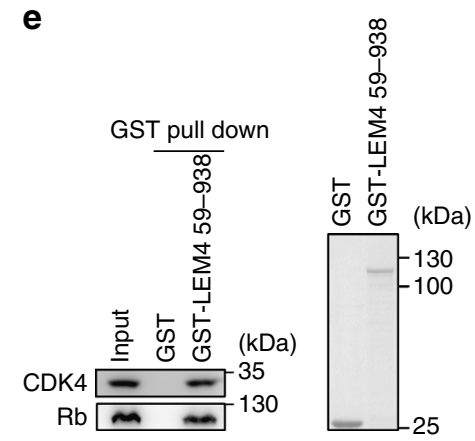

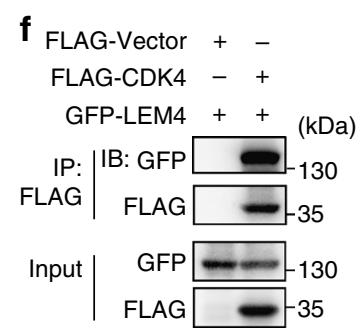

g

FLAG-Vector +-

FLAG-LEM4 - +

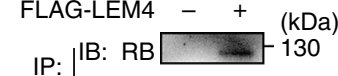

FLAG $\mid$ FLAG $\longrightarrow 130$

Input $\mid \begin{array}{r}\mathrm{RB} \sim \mathrm{F}^{130} \\ \mathrm{FLAG} \longrightarrow \mathbf{w}^{130}\end{array}$ h

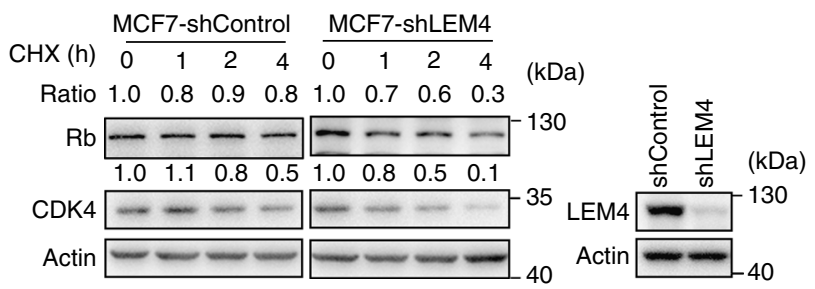

i

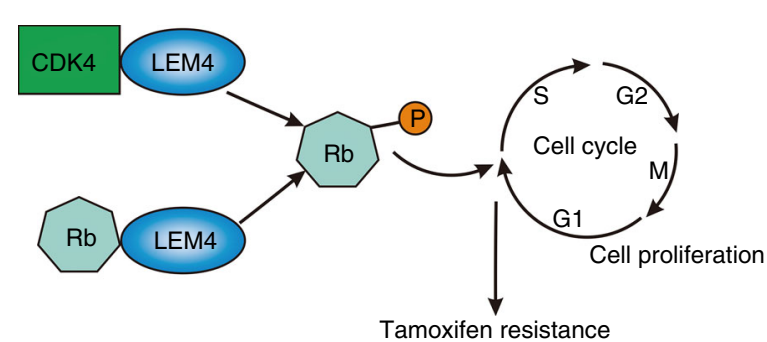

Secondly, LEM4 alters the cell cycle by promoting the G1/S phase transition. Cyclin D1, p-CDK4, and p-Rb exhibit a concerted upregulation in the LEM4 overexpressing ER+ breast cancer cells. Overexpression of LEM4 renders MCF7 cells resistant to tamoxifen, and siRNA knockdown of LEM4 or combination treatment with PD0332991 significantly overcome tamoxifen resistance among the MCF-TAMR, BT474, and MCF7-LEM4 cells. Thirdly, LEM4 not only stabilizes ERa via interaction with $\mathrm{ER} \alpha$, but also induces ERa transactivation activity. Moreover, LEM4 enhances Aurora-A-mediated phosphorylation of ERa on
Ser167. The fourth, elevated expression of LEM4 correlates with poor survival of patients with breast tumors. Data mining analysis of several GEO datasets with breast cancer patients who received systemic endocrine therapy revealed that a higher level of LEM4 was associated with poorer recurrence-free survival. Thus, LEM4 appears to be a major causal factor in endocrine therapy resistance.

Long-term endocrine treatment often leads to acquired resistance in $\mathrm{ER}+$ breast cancer. Data indicate that this may be mediated by multiple mechanisms that can potentiate cyclin 
Fig. 5 LEM4 confers tamoxifen resistance by activating the cyclin D-CDK4/6-Rb axis. a MCF7, MCF7-LEM4, and MCF7-TAMR cells were treated with 5\% DCC-FBS (vehicle), 4-OHT (1 $\mu$ M), PD0332991 (PD) $(0.2 \mu \mathrm{M})$, or a combination of 4-OHT and PD0332991. Adherent cells were tested by SRB after 9 days. Data are presented as \% parental control. Mean \pm s.d. for three independent replicates. $\mathbf{b}$ Immunoblots of lysates from cells treated as in a with indicated antibodies. c Tumor growth of MCF7-LEM4 cells as subcutaneous xenografts in athymic mice with E2 pellets when tumors reached an approximate volume of $100 \mathrm{~mm}^{3}$, then treated with tamoxifen pellet implanted subcutaneously, $100 \mathrm{mg} \mathrm{kg}^{-1}$ PD0332991 (tricubic weekly), or a combination of tamoxifen pellet and PD0332991. Mean \pm s.e.m., $n=8$. d H\&E staining and IHC for Ki-67, p-CDK4, p-Rb, and cleaved caspase 3/7 from c. Scale bars for H\&E, $150 \mu \mathrm{m}$. Scale bars for IHC, $50 \mu \mathrm{m}$. e GST alone or recombinant GST-LEM4 immobilized on glutathione-agarose beads was incubated

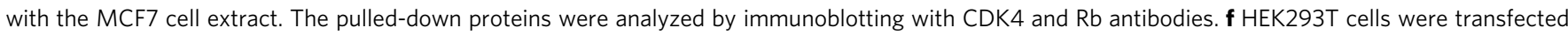
with GFP-LEM4 and pCMV6-FLAG-CDK4 or the empty vector pCMV6. The interaction of FLAG-CDK4 with GFP-LEM4 was analyzed by immunoprecipitation of the cell lysate with anti-FLAG affinity gel and immunoblotted with anti-GFP antibody. $\mathbf{g}$ HEK293T cells were transfected with pCMV6-FLAG-LEM4 or the empty vector pCMV6. The interaction of FLAG-LEM4 with Rb was analyzed by immunoprecipitation of the cell lysate with anti-FLAG affinity gel and immunoblotted with Rb antibody. h MCF7-shControl and MCF7-shLEM4 cells were treated with $50 \mu \mathrm{gL} \mathrm{mL}^{-1} \mathrm{CHX}$ for 0 , 1, 2, and $4 \mathrm{~h}$ and Western blotting was performed. $\mathbf{i}$ Model of LEM4 regulation of the cyclin D-CDK4/6-Rb axis leading to tamoxifen resistance in ER+ breast cancer. n.s., not significant. ${ }^{\star \star} P<0.01,{ }^{\star \star \star} P<0.001$. Tukey's multiple comparisons test for $\mathbf{a}, \mathbf{c}$ (weight). Repeated measures ANOVA for $\mathbf{c}$ (volume)

$\mathrm{D} 1-\mathrm{CDK} 4 / 6-\mathrm{Rb}$ signaling in an ERa-independent manner ${ }^{39-42}$. Overexpression or amplification of both cyclin D1 and CDK4 is especially high in the luminal B (58\% and $25 \%$, respectively) and HER2-enriched subtypes ( $38 \%$ and $24 \%$, respectively $)^{42}$. Consistent with these previous findings, our results reveal that patients with higher LEM4 expression have an even greater decrease in overall survival for luminal B and HER2-enriched subtypes of breast cancer. Further, LEM4 functions via a simultaneous increase in the protein levels of cyclin D1, p-CDK4, and $\mathrm{p}-\mathrm{Rb}$, each of which are reversed in LEM4-depleted cells. CDK4 activation requires both binding to cyclin D1 and its phosphorylation on Thr172 43,44 . CDK4-Thr172 phosphorylation most strongly correlates with sensitivity to PD0332991 ${ }^{43}$. We show that PD0332991 treatment results in a complete response to sensitizing MCF7-LEM4 cells to tamoxifen treatment. Furthermore, knockdown of LEM4 not only correlated with decreased p-CDK4 and $\mathrm{p}-\mathrm{Rb}$, but also restored tamoxifen sensitivity to both MCF7TAMR and BT474 cells. This functional overlap prompted our hypothesis that LEM4 acts as an A-kinase anchor protein to activate the cyclin D-CDK4-Rb signaling axis. GST-pull down assays and Co-IP studies directly support LEM4 interactions with CDK4 or Rb. The CHX chase assay suggests that LEM4 is required for $\mathrm{CDK} 4$ or $\mathrm{Rb}$ protein stabilization. Given LEM4 could coordinate the activities of VRK1 and PP2A to enable NE reassembly during mitosis ${ }^{13}$, we immunoblotted VRK1 and PP2A-C in the LEM4-depleted MCF7 cells. The results revealed that VRK1 decreased but PP2A-C elevated, and VRK1 protein degradation was independent on LEM4 (Supplementary Fig. 10a, b). VRK1 kinase has been identified as a marker for a subgroup with a poorer prognosis within the $\mathrm{ER}+$ cases $^{45-47}$. While activation of $\mathrm{PP} 2 \mathrm{~A}-\mathrm{C}$ is required for $\mathrm{E} 1 \mathrm{~A}$-mediated sensitization to drug-induced apoptosis ${ }^{48}$. Therefore, the role of LEM4 to prevent protein degradation would be target-protein dependent. Here, our data implicate LEM4 as a key regulator of the cyclin D-CDK4-Rb axis that promotes the G1/S phase transition.

Cyclin $\mathrm{D}$ is a direct transcriptional target of $\mathrm{ERa}^{49}$, and overexpression of cyclin $\mathrm{D}$ has been implicated in tamoxifen resistance ${ }^{50-52}$. Our findings showed that LEM4 induced ERa transactivation activity. Previous studies have shown that loss of ERa expression occurs in only a minority (15-20\%) of resistant breast cancers ${ }^{40}$. Here we show that LEM4 depletion aggravates ERa degradation. Overexpression of LEM4 prevents the rapid degradation of ERa in mammalian cells in an estradiol-dependent manner. Furthermore, ER $\alpha$ downregulation inhibits estrogenindependent growth of MCF7-LEM4 cells. Thus, ERa signaling might render the cell more dependent on LEM4-mediated pathways such as ERa itself. It is worthwhile to note that phosphorERa-Ser167 is one of the major mechanisms causing tamoxifen resistance ${ }^{34,36,53}$. Moreover, phosphor-ERa-Ser167 has been demonstrated to sufficiently upregulate cyclin $\mathrm{D}^{36}$. Our data show that LEM4 induces elevations of both Aurora-A and phosphorAurora-A. Aurora-A has been shown to promote distant metastases only in ER+ breast cancer cells and renders breast cancer cells resistant to tamoxifen $36,54,55$. The increased stability of Aurora-A protein results in elevated phosphor-ERa-Ser167 levels. Collectively, these findings provide an underlying mechanism for LEM4 activation of ERa signaling and contribution to increased cyclin D1 expression. In addition, overexpression of HER2 is one of the best characterized mechanisms of endocrine resistance, our data implicate LEM4, might like emerin ${ }^{56}$, act as a downstream effector for HER2 signaling pathway. Exploration results of dataset GSE33658, which based on a phase II neoadjuvant trial of anastrozole (A), fulvestrant $(\mathrm{F})$, and gefitinib $(\mathrm{G})$ in patients with ER + breast cancer, showed that decreased expression of LEM4 was associated with complete response or partial response to both AF-treatment and AFG-treatment. Thus, functional antagonism of LEM4 might allow the attack of multiple therapeutic targets simultaneously in breast cancer.

In summary, our study presents a more integrated visual of how LEM4 proteins orchestrate the major group of molecules controlling the cyclin D-CDK4-Rb axis activated during the G1/S phase transition. Moreover, we present evidence for LEM4 acting as a scaffold for both Aurora-A and ERa and promoting the activation of ERa signaling. The activated cyclin D-CDK4/6-Rb signaling and ERa signaling subsequently drive the transition of breast cancer cells to estrogen independence and tamoxifen resistance. Further study is needed to characterize the functions of the interaction of LEM4 proteins with mitotic kinases, such as CDK1, Aurora-A, and Aurora-B, during tumorigenesis and metastasis.

\section{Materials and methods}

Cell lines and cell culture. The human breast cancer cell lines MCF7, T47D, BT474, and MDA-MB-231 were purchased from American Type Cell Culture (Manassas, VA) and cultured in DMEM or RPMI-1640 medium supplemented with $10 \%$ fetal bovine serum (FBS). The MCF7-TAMR cell model was kindly provided by Dr Tao Zhu (University of Science and Technology of China) ${ }^{57,58}$. All cells were maintained at $37^{\circ} \mathrm{C}$ with $5 \% \mathrm{CO}_{2}$. For deriving vector-control and LEM4-overexpression cell lines, pCMV6 vector or pCMV6-3×FLAG-LEM4 was stably transfected into MCF7 and T47D cells using Lipofectamine 2000 (Invitrogen, Thermo Fisher Scientific). To generate LEM4 shRNA cells, two different shRNA hairpins specifically targeting human LEM4 (Supplementary Table 1) were cloned into LKO.1 and used to knock down LEM4 constitutively in various breast cancer cell lines. All cell lines had been authenticated by STR profiling analysis.

Construction of expression plasmids. The pCMV6-FLAG-LEM4 and pCMV6FLAG-CDK4 plasmids were purchased from OriGene. Mammalian expression plasmids for GFP-Aurora-A and GFP-ERa were generated in our laboratory. The mammalian expression plasmids for various ERa mutants were constructed by PCR amplification using the primers listed in Supplementary Table 2. The bacterial 
a

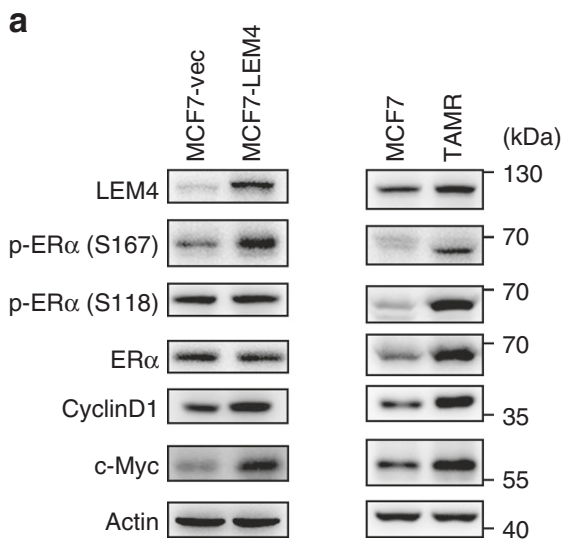

b

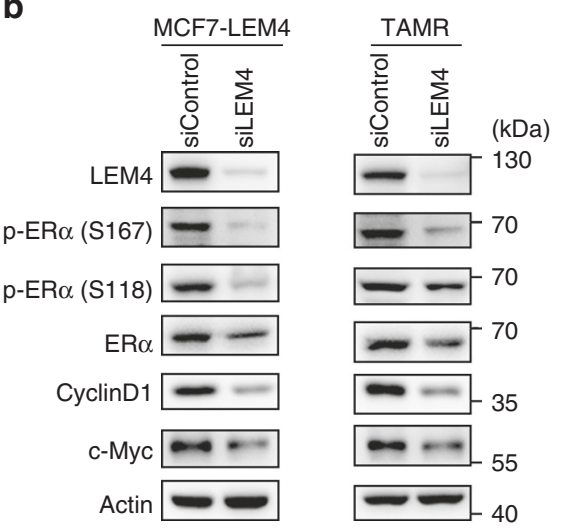

C

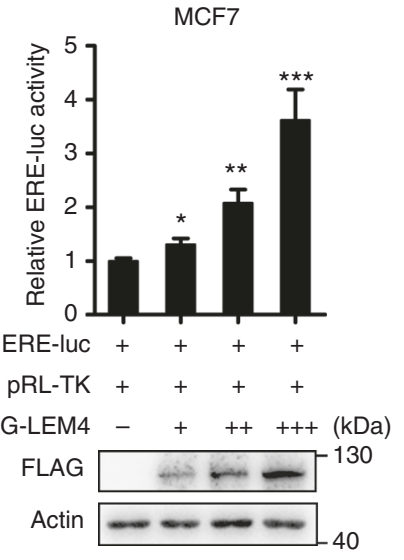

d

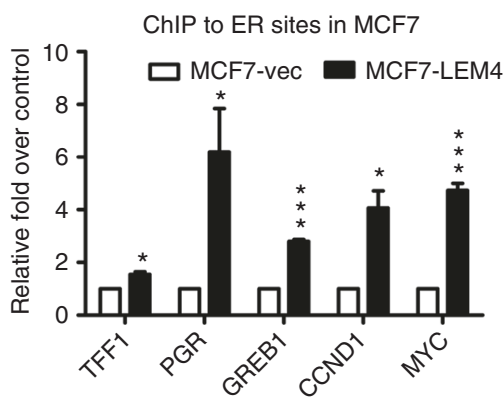

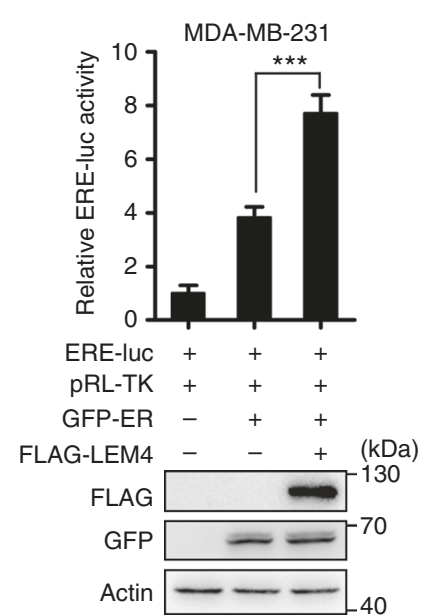

e
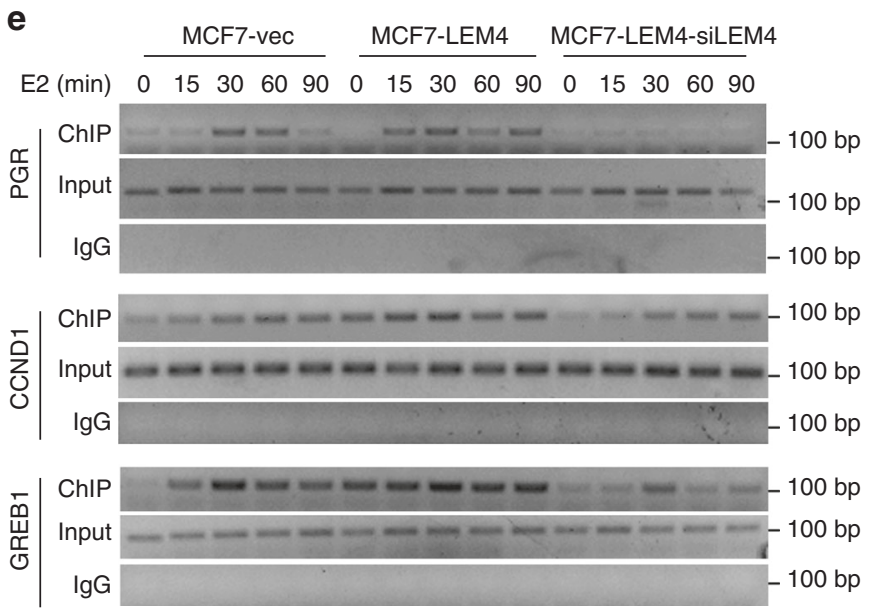

Fig. 6 LEM4 induces ER $\alpha$ transactivation activity. a Immunoblot analysis of the phosphorylation of ER, Cyclin D1, and C-Myc in MCF-LEM4 and MCF7TAMR cells. b Immunoblot analysis of the phosphorylation of ER, Cyclin D1, and c-Myc in LEM4-depleted MCF7-LEM4 and LEM4 knocked-down MCF7TAMR cells. c Luciferase assay. ER+ MCF7 and ER $\alpha$-negative MDA-MB-231 cells were transfected with ERE-Luc and other indicated plasmids. Following incubation for $48 \mathrm{~h}$, luciferase activity was measured and normalized to Renilla. Results are the mean \pm s.e.m. of three independent experiments performed in triplicate. $\mathbf{d}$ ER $\alpha$ ChIP assay of known ER-binding sites in ER $\alpha$ target genes was performed in MCF7-LEM4 cells incubated in estrogen-depleted medium ( $5 \%$ charcoal-stripped serum in phenol red-free DMEM) for $72 \mathrm{~h}$ before treatment with vehicle, $10 \mathrm{nmol} \mathrm{L}{ }^{-1} \mathrm{E} 2$ for 45 min. e Time course ChIP study of the endogenous ER $\alpha$ with the estrogen response elements in the promoter region of the ER $\alpha$ target genes. MCF7, MCF7-LEM4, and LEM4-depleted MCF7LEM4 cells incubated in estrogen-depleted medium (5\% charcoal-stripped serum in phenol red-free DMEM) for $72 \mathrm{~h}$ before treatment with vehicle, $10 \mathrm{nmol} \mathrm{L}^{-1} \mathrm{E2}$. ChIP analysis was conducted by using anti-ER $\alpha$ antibody. ${ }^{\star} P<0.05,{ }^{\star \star} P<0.01,{ }^{\star \star \star} P<0.001$. Tukey's multiple comparisons test for c. Student's t-test for $\mathbf{d}$ 
a

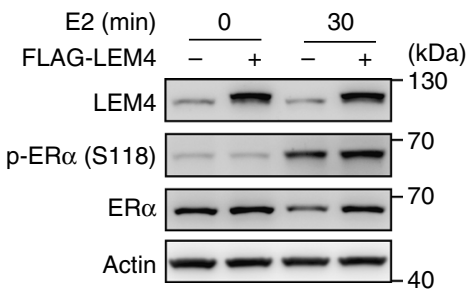

C

IP

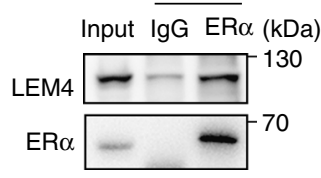

d
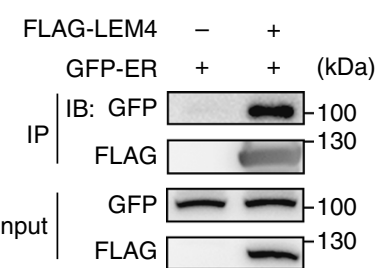

f
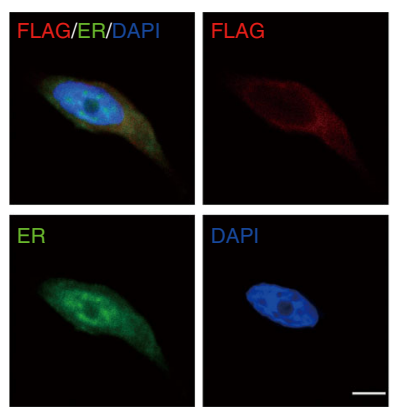

$\mathbf{g}$

MCF7-vec MCF7-LEM4

siCtl ++-++-

SiESR1 - $-+-\ldots+$

Fulvestrant $-+-\quad-+-\quad(\mathrm{kDa})$

$\mathrm{ER} \alpha \backsim$

Actin $--\longrightarrow-40$

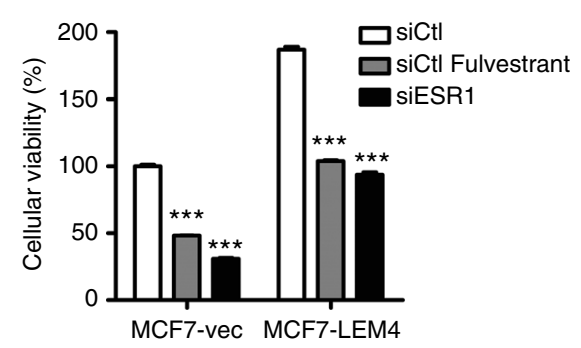

b

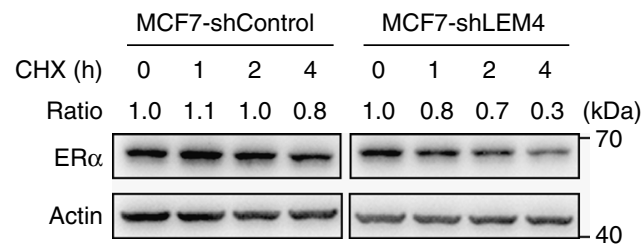

e
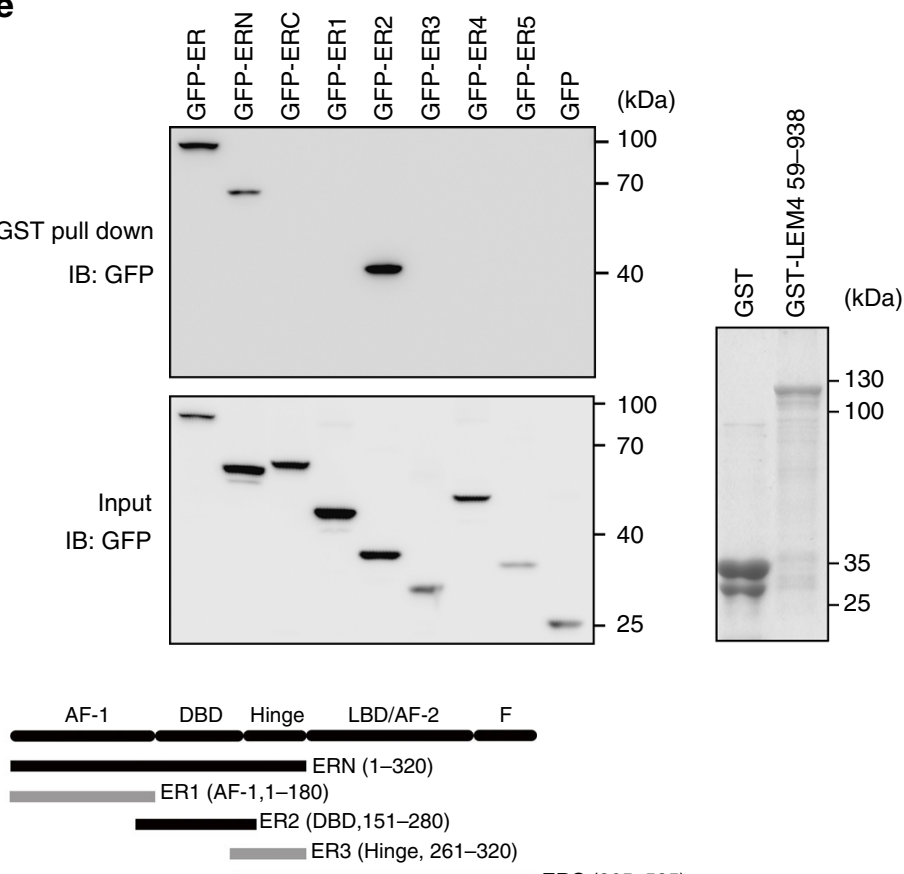

ERC (265-595)

ER4 (LBD/AF-2, 321-550)

ER5 ((F, 500-595)

h

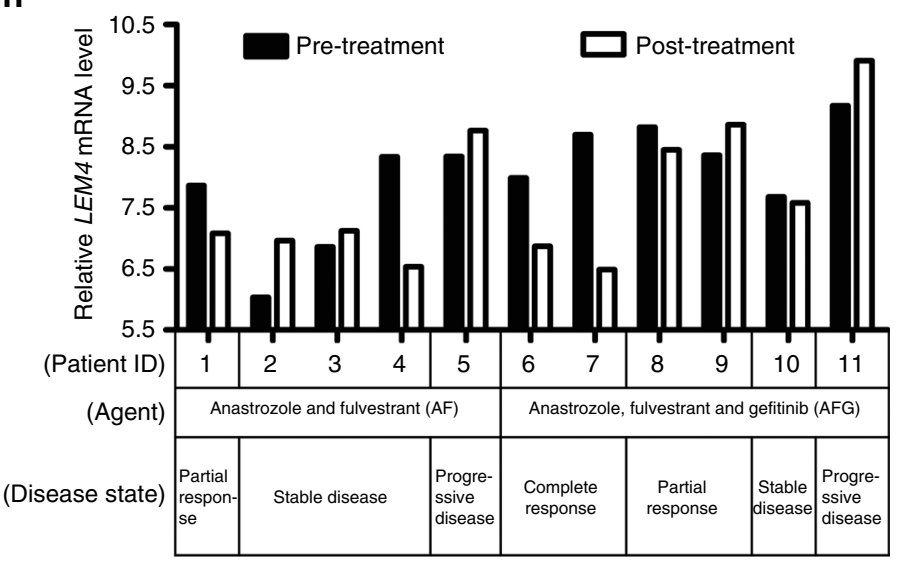

expression plasmids for GST-LEM4 (containing amino acids 59-938) were constructed by insertion of the cDNA fragments into the pGEX-6P-1 vector.

Breast cancer molecular subtype and survival analysis. Cancer subtype-specific LEM4 gene expression analysis was performed on TCGA_BRCA_exp_HiSeqV22015-02-24 mRNA expression data. The mRNA-scores were normalized and expressed as a $Z$-scores following the formula $Z=$ (LEM4_score of each sample mean LEM4_score)/(s.d. of all samples). Kaplan-Meier survival analysis was performed using an online database (www.kmplot.com) and the data was analyzed using median cutoff in each case. The study survival curves based on the LEM4 protein expression score in 284 patients were plotted using Kaplan-Meier analysis and the statistical parameters calculated by log-rank (Mantel-Cox) test using GraphPad Prism 6 software.

Cell proliferation and viability assays. Cell proliferation was monitored by SRB assay and EdU (5-ethynyl-2'-deoxyuridine) incorporation assay. The SRB assay was performed following an already established procedure ${ }^{59}$. Briefly, cells were plated into 24 -well plates, followed by incubation of cells with treatment of 
Fig. 7 LEM4 interacts with and stabilizes ER $\alpha$. a Immunoblot analysis of ER $\alpha$ in MCF7 and MCF7-LEM4 cells grown under estrogen-deprived conditions in DMEM phenol-free medium containing 5\% dextran charcoal-stripped serum and treated with $10 \mathrm{nmol} \mathrm{L}^{-1}$ E2 for 30 min. b MCF7-shControl and MCF7shLEM4 cells were treated with $50 \mu \mathrm{g} \mathrm{mL}{ }^{-1} \mathrm{CHX}$ for $0,1,2$, and $4 \mathrm{~h}$ and ER $\alpha$ analyzed by immunoblot. c For endogenous LEM4 and ER $\alpha$ interaction, BT474 cells were immunoprecipitated with anti-ER $\alpha$ antibody and detected with anti-LEM4 antibody. d HEK293T cells were transfected with FLAG-LEM4 and GFP-ER $\alpha$ or the empty vector pCMV6. After incubation for $48 \mathrm{~h}$, cell lysates were precipitated with anti-FLAG affinity gel and immunoblotted with anti-GFP and anti-FLAG antibody. e GST alone or GST-LEM4 immobilized on glutathione-agarose beads was incubated with the cell extract of HEK293T cells transfected with GFP-ER $\alpha$ or various mutants of ER $\alpha$ tagged with GFP. Bound proteins were separated by SDS-PAGE and immunoblotted with anti-ER $\alpha$ antibody. $\mathbf{f}$ MCF7-LEM4 cells were immunostained with anti-FLAG (indicated LEM4, red) and anti-ER (green) antibody, and counterstained with DAPI (blue). Scale bars, $7.5 \mu \mathrm{m}$. g MCF7-LEM4 cells were transfected with siRNA of ESR1 or treated with fulvestrant for 6 days. Total cell viability were assessed by SRB assays. Results are the mean $\pm \mathrm{s}$. d. of three independent experiments performed in triplicate. Western blot was performed with anti-ER $\alpha$ antibody. h Compared the relative mRNA LEM4 level between pre-treatment and post-treatment in samples from GEO GSE33658. ${ }^{\star \star \star} P<0.001$. Tukey's multiple comparisons test for $\mathbf{g}$

choice for different times, cell fixation and SRB staining, and absorbance measurement. The EdU incorporation assay was performed according to the manufacturer's instructions (EdU- assay kit, Beyotime Biotechnology, Shanghai, China). Briefly, cells were cultured in 24-well plates and $50 \mu \mathrm{M}$ EdU added to each well. The cells were cultured for an additional $2 \mathrm{~h}$. Cells were subsequently fixed on glass coverslips with $4 \%$ paraformaldehyde before undergoing Apollo staining for $30 \mathrm{~min}$ and Hoechst 33342 staining for $30 \mathrm{~min}$. The EdU incorporation rate was expressed as the ratio of EdU-positive cells to total Hoechst-positive cells. Experiments were performed in triplicate.

For cell cycle analysis, cells were collected and washed twice with ice-cold phosphate-buffered saline (PBS). The cells were fixed with $70 \%$ ethanol at $4{ }^{\circ} \mathrm{C}$ for $24 \mathrm{~h}$ and incubated with 4',6-diamidino-2-phenylindole (DAPI) (Sigma-Aldrich, St. Louis, Missouri) for $10 \mathrm{~min}$. Samples were analyzed using Millipore Amnis ${ }^{\circledR}$ Imaging Flow Cytometers (EMD Millipore, Darmstadt, Germany).

For cell viability assays, cells were plated at $2 \times 10^{4}$ cells per well in 24 -well plates, in triplicate, in the presence of $5 \%$ dextran-charcoal-treated FBS (DCC-FBS) with 4-OHT, PD0332991 treatments at stated concentrations, a combination of the two, or vehicle for 9 days. Cell viability was measured by SRB assay. Triplicates were averaged for mean absorbance, and a percentage calculated for the survival of drug-treated cells versus time-matched vehicle-treated cells. Experiments were performed in triplicate.

Transwell invasion assay. The Boyden chamber assay was used for invasion assay. Briefly, $1 \times 10^{5}$ cells suspended in $200 \mu \mathrm{l}$ serum-free medium were plated into the top chamber with $50 \mu$ l growth factor reduced Matrigel-coated membrane ( $8 \mu \mathrm{M}$ pole size, BD Biosciences, Shanghai, China). The chambers were then placed into 24-well plates with $600 \mu \mathrm{l}$ serum-containing (10\%) medium in each well. After $24 \mathrm{~h}$ incubation, cells on the bottom side of the chamber membrane were fixed, stained with crystal violet and photographed.

Soft agar colony formation assay. The MCF7 and T47D cell lines and their derived cell lines were cultured in DMEM or RPMI-1640/ with 10\% FBS in 6-well plates within a $0.35 \%$ agar layer, and $2 \times 10^{3}$ cells were seeded to the middle layer of the soft agar (Lonza, Rockland, USA). The plates were incubated for 14 days (T47D) or 21 days (MCF7), after which the cultures were inspected and photographed. Assays were conducted in triplicate in a single experiment, and then as three independent experiments.

3D matrigel assay. Briefly, the 8-well chamber slide (BD Bioscience, catalog number: 354108 ) is precoated with $80 \mu$ growth factor reduced BD Matrigel ${ }^{\mathrm{TM}}$ Matrix (BD Biosciences, catalog number: 354230) per well. Then the chamber slide is transferred to a cell culture incubator to allow matrigel solidification for at least $15 \mathrm{~min}$. Five thousand cells for MCF-10A in assay medium containing $5 \mathrm{ng} \mathrm{ml}^{-1}$ EGF and 2\% Matrigel were seeded in each well. Medium were replenished every 3 days. Images of spheres with defined scales were subjected to the ImageJ computer program to determine the area covered by each sphere, and the diameter of that sphere was then calculated based on the circle formula.

Luciferase reporter assay. MCF7 cells were transiently transfected with EREaLuc, pCMV6-FLAG-LEM4, or pCMV6-FLAG and pRL-TK (Renilla luciferase, Promega) as an internal control. MDA-MB-231 cells were transiently transfected with EREa-Luc, wild-type ERa, pCMV6-FLAG-LEM4, or pCMV6-FLAG and pRLTK. After $48 \mathrm{~h}$ of transfection, luciferase activity was measured using a luminometer (Tristar LB941, Berthold Technologies, BadWild, Germany). Firefly luciferase activity was normalized to the Renilla luciferase activities. Experiments were performed in triplicate.

Chromatin immunoprecipitation. MCF7 cells, MCF7-LEM4 cells, and MCF7LEM4 cells with depletion of LEM4 expression by siRNA, grown for 3 days in phenol red-free DMEM supplemented with 5\% charcoal-treated FBS. Cells were treated with either vehicle or $10 \mathrm{nM}$ E2 for various time. The Simple ChIP ${ }^{\circledR}$ Enzymatic Chromatin IP Kit (Cell Signaling, catalog number: \#9003) was used to perform the chromatin immunoprecipitation (ChIP) assay according to the manufacturer's instruction. Briefly, cells were cross-linked with $1 \%$ formaldehyde for $10 \mathrm{~min}$ at room temperature. Glycine quenched samples were washed with icecold PBS, collected, and then nuclei were collected after cell lysis. Micrococcal nuclease was added to the nuclei suspension to digest the DNA for $20 \mathrm{~min}$ at $37^{\circ} \mathrm{C}$ Subsequently, the digest reactions were stopped by the addition of $0.5 \mathrm{M}$ EDTA. Nuclear pellet were collected and were incubated in ChIP buffer with protease inhibitors for $10 \mathrm{~min}$ on ice. Sheared cross-linked-chromatin preparation was collected after sonication. Chromatin extracts containing DNA fragments of 150-900 base pairs were immunoprecipitated using anti-ER $\alpha$ or anti-IgG antibody. Quantitative real-time PCR analyses were performed using the Realplex real-time PCR detection system (Eppendorf). The sequences of the primers described in Supplementary Table 4.

RNA interference. SiRNA oligos for RNAi against human LEM4, AKT, and Aurora-A were synthesized by Invitrogen (Supplementary Table 1) and transfected using the Lipofectamine RNAiMAX reagent according to the manufacturer's instructions (Invitrogen).

RNA extraction and real-time RT-PCR. Total RNA was isolated using Trizol reagent (Roche Diagnostics, Indianapolis, Indiana) according to the manufacturer's protocol. A total of $2 \mu \mathrm{g}$ of RNA was reverse-transcribed using the PrimeScript ${ }^{\mathrm{m}} \mathrm{RT}$ reagent Kit with gDNA Eraser (TaKaRa). The SYBR green (TaKaRa) method was used with the Realplex real-time PCR detection system (Eppendorf) to detect gene expression. Real-time PCR was performed in triplicate. The sequences of the oligonucleotide primers used for real-time PCR are described in Supplementary Table 3.

Western blot analysis. The cells were collected and resuspended in cell lysis buffer containing $50 \mathrm{mM}$ Tris ( $\mathrm{pH}$ 7.4), $150 \mathrm{mM} \mathrm{NaCl}, 1 \mathrm{mM}$ EDTA, 1 mM EGTA, $1 \mathrm{mM} \mathrm{NaF}, 1 \mathrm{mM}$ Na3VO4, $1 \%$ Triton X-100, 10\% glycerol, $0.25 \%$ deoxycholate, and $0.1 \%$ SDS. Lysates were electrophoresed using SDS-PAGE and blotted onto nitrocellulose (NC) membrane. Membranes were blocked with $5 \%$ nonfat milk or $5 \%$ BSA solution for $2 \mathrm{~h}$. Samples were probed with primary antibodies overnight at $4{ }^{\circ} \mathrm{C}$ (for antibody details, see Supplementary Table 5). Secondary antibodies HRP conjugated donkey anti-Rabbit IgG (GE Healthcare NA934V) or goat antimouse IgG $(\mathrm{H}+\mathrm{L})(\mathrm{ZB} 2305)$ were diluted at 1:5000. Blots were photographed by the Image Quant LAS 4000 luminescent image analyzer (General Electric, Fairfield, CT). All Western blots were quantified using the Image J program (NIH, USA). Uncropped scans can be found in Supplementary Fig. 11, 12, 13, 14, 15.

Immunofluorescence staining. Cells were fixed with 4\% paraformaldehyde for 30 min, permeabilized with $0.5 \%$ (vol/vol) Triton X-100 for $20 \mathrm{~min}$ and blocked with $10 \%$ normal goat serum in phosphate-buffered saline for $30 \mathrm{~min}$ at room temperature. Cells were then incubated with primary antibodies (E-cadherin, 1:1000; ERa, 1:100; FLAG, 1:400) overnight at $4{ }^{\circ} \mathrm{C}$. After washing, cells were incubated with secondary antibodies conjugated with FITC (anti-rabbit antibody, 1:1000) or RITC (anti-mouse antibody, 1:500) at room temperature for $2 \mathrm{~h}$ and washed three times with PBS. Nuclei were stained with DAPI (4',6-diamidino-2-phenylindole) for $10 \mathrm{~min}$. The fluorescence images were taken with confocal microscope (Leica TCS SP5, Germany).

Immunoprecipitation and GST pull-down assay. For immunoprecipitation, cells were transfected with pCMV6-FLAG-LEM4 or the empty vector and the cell lysate incubated with anti-FLAG-agarose beads at $4{ }^{\circ} \mathrm{C}$ for $2 \mathrm{~h}$ (Sigma). The beads were washed extensively and eluted under native conditions by competition with $3 \times$ FLAG peptide (Sigma). The supernatants were analyzed by Western blotting as 
a

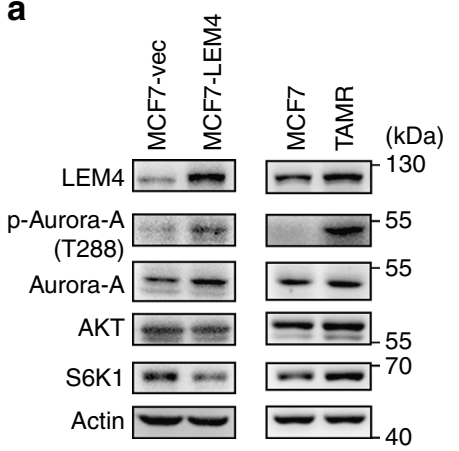

b

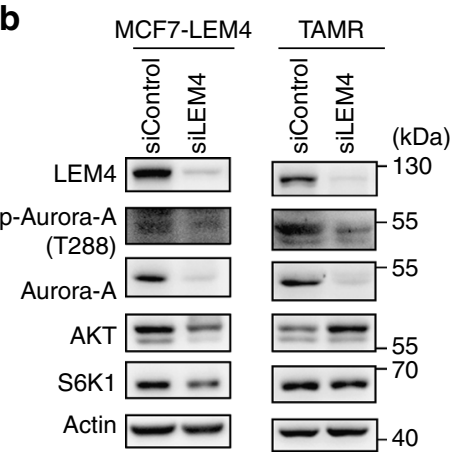

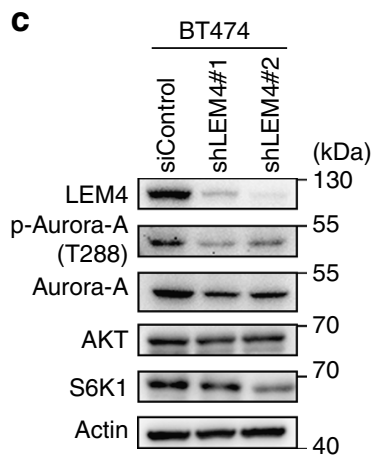

d

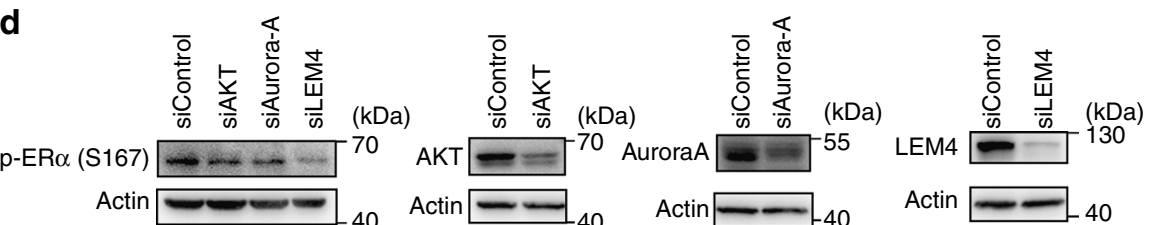

e

f
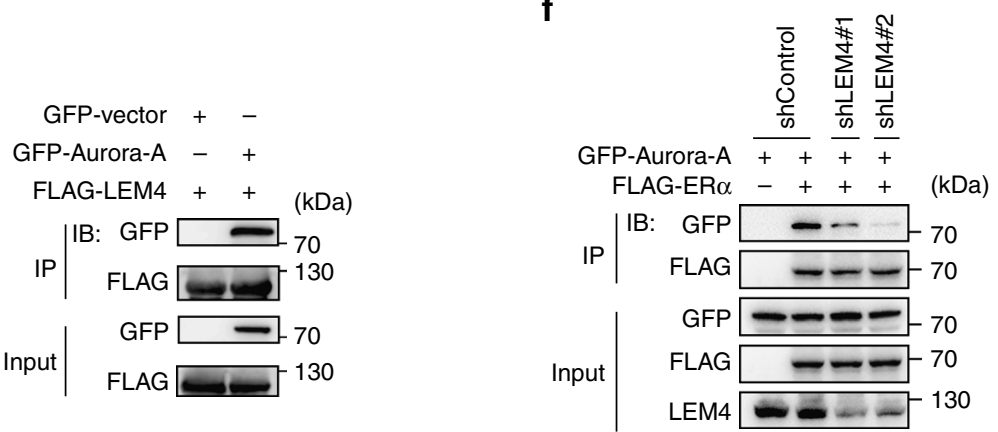

g

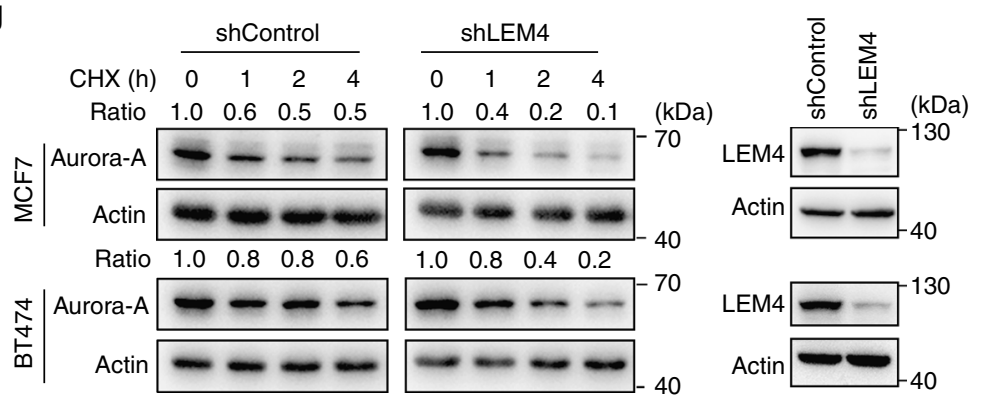

h

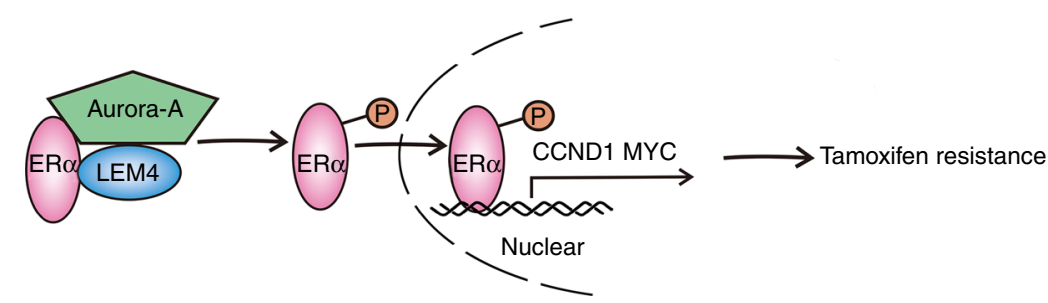

Fig. 8 LEM4 mediated the phosphorylation of ER $\alpha$-Ser167 by Aurora-A. a Immunoblot analysis of the phosphorylation of Aurora-A, Aurora-A, AKT, and S6K1 in MCF7-LEM4 and MCF7-TAMR cells. b LEM4 siRNA-treated and control siRNA-treated MCF7-LEM4 and MCF7-TAMR cells were treated for 48 h. Western blot was performed with indicated antibodies. c BT474 cells were transfected with LEM4 siRNA or control siRNA for $48 \mathrm{~h}$. Western blot was performed with indicated antibodies. d MCF7-LEM4 cells were transfected with LEM4 siRNA or AKT siRNA or Aurora-A siRNA for $48 \mathrm{~h}$. Western blot was performed with indicated antibodies. e HEK293T cells were transfected with FLAG-LEM4 and GFP-Aurora-A. After incubating for $48 \mathrm{~h}$, cell lysates were precipitated with anti-FLAG affinity gel and immunoblotted with anti-GFP antibody. $\mathbf{f}$ HEK293T-shLEM4 cells were transfected with FLAG-ER $\alpha$ and GFPAurora-A. After incubating for $48 \mathrm{~h}$, cell lysates were precipitated with anti-FLAG affinity gel and immunoblotted with anti-GFP antibody. $\mathbf{g}$ MCF7shControl and MCF7-shLEM4 cells, or BT474-shControl and BT474-shLEM4 cells were treated with $50 \mu \mathrm{g} \mathrm{mL}{ }^{-1} \mathrm{CHX}_{\text {for }} 0,1,2$, and $4 \mathrm{~h}$ and analyzed for Aurora-A by immunoblot. $\mathbf{h}$ Model of LEM4 regulation of the ER $\alpha$ signaling leading to tamoxifen resistance in ER+ breast cancer 
described above. For GST pull-down assays, the GST alone or recombinant GSTLEM4 immobilized on glutathione-agarose beads was incubated with the MCF7 cell extract at $4^{\circ} \mathrm{C}$ for $2 \mathrm{~h}$. The beads were washed extensively and the pulled-down proteins mixed with SDS-PAGE sample buffer for analysis by immunoblotting with appropriate antibodies.

Mouse xenograft studies. All animal studies were approved by the Institutional Animal Care and Use Committee at Nankai University. For Fig. 2f, g, estrogen pellets (60-day slow release pellet containing $0.72 \mathrm{mg}$; Innovative Research of America) were implanted subcutaneously at the nape of the neck of female 3 to 4 week-old $\mathrm{BALB} / \mathrm{c} \mathrm{nu} / \mathrm{nu}$ athymic mice (Vitalriver Beijing, China). Three days later, $5 \times 10^{6}$ MCF7-vec/MCF7-LEM4 or T47D-vec/T47D-shLEM4 cells were suspended in $100 \mu \mathrm{L}$ of PBS/Matrigel (1:1) (BD Biosciences, Franklin Lakes, New Jersey) and injected subcutaneously. For Supplementary Figure 5b, $5 \times 10^{6}$ MCF7-vec/MCF7LEM4 cells were suspended in $100 \mu \mathrm{L}$ of PBS/Matrigel (1:1) (BD Biosciences) and injected subcutaneously into the nude mice without estrogen pellet implantation. For Fig. 4f, g, $5 \sim 8 \times 10^{6}$ cells $\left(5 \times 10^{6}\right.$ MCF7 cells and $8 \times 10^{6}$ BT474 cells $)$ suspended in $100 \mu \mathrm{L}$ of PBS/Matrigel (1:1) were injected subcutaneously into female nude mice with the estrogen pellets implanted 3 days prior. For MCF7 cells in Fig. 4 f, when the tumor size reached $\sim 100 \mathrm{~mm}^{3}$, seven mice in each group were treated with or without tamoxifen pellets implanted subcutaneously (60-day slow release pellet containing $5 \mathrm{mg}$; Innovative Research of America). For BT474 cells in Fig. $4 \mathrm{~g}$, mice were treated with tamoxifen pellets implanted subcutaneously and when the tumor size reached $\sim 200 \mathrm{~mm}^{3}$. For Figs $5 \mathrm{c}, 1 \times 10^{7}$ cells suspended in $100 \mu \mathrm{L}$ of PBS/Matrigel (1:1) were injected subcutaneously into the mammary fat pads of female nude mice with the estrogen pellets implanted 3 days prior. When the tumor size reached $\sim 200 \mathrm{~mm}^{3}$, the mice were randomized into three groups and treated with subcutaneous tamoxifen pellet implants, $100 \mathrm{mg} \mathrm{kg}^{-1}$ PD0332991 (MedChem Express) tricubic weekly by intragastric-administration for 6 weeks, or a combination of tamoxifen and PD0332991. After 8 to 10 weeks, mice were euthanized and the tumors were sectioned and fast-frozen, or formalin-fixed and paraffin-embedded, and H\&E-stained slides made. The tumors were measured using a Vernier caliper and the tumor volume was estimated as follows: $V=$ (length $\times$ width $\times$ height $\times 0.5) \mathrm{mm}^{3}$.

IHC in xenograft tumors. Tissue sections of xenograft tumor (4- $\mu \mathrm{m}$-thick) were immunostained with anti-LEM4 (1:500), anti-Ki-67 (1:500), anti-p-CDK1 (1:500), anti-p-CDK4 (1:500), anti-cyclin D1 (1:2000), anti-p-Rb (1:500), anti-caspase 3/7 (1:500), or anti-p-ERa167 (1:500) antibodies overnight at $4{ }^{\circ} \mathrm{C}$. The peroxidaseconjugated streptavidin complex method was performed, followed by the $3,3^{\prime}$ diaminobenzidine $(\mathrm{DAB})$ procedure according to the manufacturer's protocols (Dako, Agilent pathology solutions).

IHC for tissue microarrays. Breast cancer tissue microarray slides (HBreDuc150Sur-01/02) were purchased from Shanghai Outdo Biotech Co. Ltd. (SOBC). All patients gave consent for the use of their tissue samples and clinical data. The ethic review board at the Tianjin Central Hospital of Gynecology Obstetrics approved the study. Staining of the tissue microarray was performed according to previously described protocols ${ }^{60}$. Briefly, after deparaffinization in xylene and rehydration in a series of alcohols (100-75\%), slides were incubated in the dual endogenous enzyme block (Dako, Carpinteria, CA) for $15 \mathrm{~min}$ to inactivate endogenous peroxide activity and treated in citrate buffer $(\mathrm{pH} \mathrm{6.0)}$ for $3 \mathrm{~min}$ in a pressure cooker for antigen retrieval. After cooling for $45 \mathrm{~min}$ at room temperature, slides were incubated with rabbit anti-LEM4 antibody at $1: 400$ dilution at $4{ }^{\circ} \mathrm{C}$ overnight and with the secondary horseradish peroxidase-labeled polymer antirabbit IgG for $30 \mathrm{~min}$ at room temperature. With diaminobenzidine tetrahydrochloride (Dako) as a chromogen, slides were counter stained with hematoxylin. Slides were scanned at $20 \times$ using the Aperio ScanScope XT pathology system (Leica Microsystems, Germany). Images were exported to Aperio ImageScope for viewing. The LEM4 staining score was calculated according to the following procedures. The intensity of staining $(0=$ negative, $1=$ low, $2=$ medium and $3=$ high $)$ and the percentage of positively stained cells $(0-100 \%)$ were recorded for each specimen, and the LEM4 expression score was calculated as intensity score $\times$ percentage of positive cells $\times 100$. In order to translate continuous LEM4 expression into a clinical decision, the median cutoff point was used to stratify patients into two groups.

Statistical analysis. All in vitro experiments were repeated at least three times unless stated otherwise. Student's $t$-test or one-way ANOVA for multiple group comparisons were performed using SPSS 21 . Survival curves were plotted using Kaplan-Meier analysis and the statistical parameters calculated by a log-rank test using GraphPad Prism 6 software. All statistical tests were two-sided, and differences were considered statistically significant at $P<0.05$ unless stated otherwise.

\section{Data availability}

The data that support the findings of this study are available from the corresponding author upon reasonable request. The URL was provided for each of GEO datasets, which was obtained from Gene Expression Omnibus (GEO) database (https://www.ncbi.nlm. nih.gov/geo). GSE2990, GSE3494, GSE9195, GSE33658, GSE2034, GSE16446, GSE20685, GSE100075.

Received: 8 January 2018 Accepted: 2 July 2018

Published online: 09 October 2018

\section{References}

1. Early Breast Cancer Trialists' Collaborative G. Effects of chemotherapy and hormonal therapy for early breast cancer on recurrence and 15 -year survival: an overview of the randomised trials. Lancet 365, 1687-1717 (2005).

2. Murphy, C. G. \& Dickler, M. N. Endocrine resistance in hormone-responsive breast cancer: mechanisms and therapeutic strategies. Endocr. Relat. Cancer 23, R337-R352 (2016)

3. Cristofanilli, M. et al. Fulvestrant plus palbociclib versus fulvestrant plus placebo for treatment of hormone-receptor-positive, HER2-negative metastatic breast cancer that progressed on previous endocrine therapy (PALOMA-3): final analysis of the multicentre, double-blind, phase 3 randomised controlled trial. Lancet Oncol. 17, 425-439 (2016).

4. DeMichele, A. et al. CDK 4/6 inhibitor palbociclib (PD0332991) in Rb+ advanced breast cancer: phase II activity, safety, and predictive biomarker assessment. Clin. Cancer Res. 21, 995-1001 (2015).

5. Finn, R. S. et al. Palbociclib and Letrozole in Advanced Breast Cancer. N. Engl. J. Med. 375, 1925-1936 (2016).

6. Sherr, C. J., Beach, D. \& Shapiro, G. I. Targeting CDK4 and CDK6: from discovery to therapy. Cancer Discov. 6, 353-367 (2016).

7. Bussolati, G. Proper detection of the nuclear shape: ways and significance. Rom. J. Morphol. Embryol. 49, 435-439 (2008).

8. Bussolati, G., Marchio, C., Gaetano, L., Lupo, R. \& Sapino, A. Pleomorphism of the nuclear envelope in breast cancer: a new approach to an old problem. $J$. Cell. Mol. Med. 12, 209-218 (2008).

9. Denais, C. M. et al. Nuclear envelope rupture and repair during cancer cell migration. Science 352, 353-358 (2016).

10. Lim, S., Quinton, R. J. \& Ganem, N. J. Nuclear envelope rupture drives genome instability in cancer. Mol. Biol. Cell 27, 3210-3213 (2016).

11. Raab, M. et al. ESCRT III repairs nuclear envelope ruptures during cell migration to limit DNA damage and cell death. Science 352, 359-362 (2016)

12. Lin, F. et al. MAN1, an inner nuclear membrane protein that shares the LEM domain with lamina-associated polypeptide 2 and emerin. J. Biol. Chem. 275, 4840-4847 (2000)

13. Asencio, C. et al. Coordination of kinase and phosphatase activities by Lem 4 enables nuclear envelope reassembly during mitosis. Cell 150, 122-135 (2012).

14. Ulbert, S., Antonin, W., Platani, M. \& Mattaj, I. W. The inner nuclear membrane protein Lem2 is critical for normal nuclear envelope morphology. FEBS Lett. 580, 6435-6441 (2006).

15. Antoniou, A. C. et al. A locus on 19p13 modifies risk of breast cancer in BRCA1 mutation carriers and is associated with hormone receptor-negative breast cancer in the general population. Nat. Genet. 42, 885-892 (2010).

16. Brachner, A. \& Foisner, R. Lamina-associated polypeptide (LAP)2alpha and other LEM proteins in cancer biology. Adv. Exp. Med. Biol. 773, 143-163 (2014).

17. Chow, K. H., Factor, R. E. \& Ullman, K. S. The nuclear envelope environment and its cancer connections. Nat. Rev. Cancer 12, 196-209 (2012).

18. Thul, P. J. et al. A subcellular map of the human proteome. Science 356, https://doi.org/10.1126/science.aal3321 (2017).

19. Wang, Y. et al. Gene-expression profiles to predict distant metastasis of lymph-node-negative primary breast cancer. Lancet 365, 671-679 (2005).

20. Sotiriou, C. et al. Gene expression profiling in breast cancer: understanding the molecular basis of histologic grade to improve prognosis. J. Natl Cancer Inst. 98, 262-272 (2006).

21. Desmedt, C. et al. Multifactorial approach to predicting resistance to anthracyclines. J. Clin. Oncol. 29, 1578-1586 (2011).

22. Kao, K. J., Chang, K. M., Hsu, H. C. \& Huang, A. T. Correlation of microarray-based breast cancer molecular subtypes and clinical outcomes: implications for treatment optimization. Bmc. Cancer 11, 143 (2011).

23. Hanahan, D. \& Weinberg, R. A. Hallmarks of cancer: the next generation. Cell 144, 646-674 (2011).

24. Musgrove, E. A. \& Sutherland, R. L. Biological determinants of endocrine resistance in breast cancer. Nat. Rev. Cancer 9, 631-643 (2009).

25. Martin, L. A. et al. Discovery of naturally occurring ESR1 mutations in breast cancer cell lines modelling endocrine resistance. Nat. Commun. 8, 1865 (2017).

26. Miller, L. D. et al. An expression signature for p53 status in human breast cancer predicts mutation status, transcriptional effects, and patient survival. Proc. Natl Acad. Sci. USA 102, 13550-13555 (2005). 
27. Loi, S. et al. PIK3CA mutations associated with gene signature of low mTORC1 signaling and better outcomes in estrogen receptor-positive breast cancer. Proc. Natl Acad. Sci. USA 107, 10208-10213 (2010).

28. Doisneau-Sixou, S. F. et al. Estrogen and antiestrogen regulation of cell cycle progression in breast cancer cells. Endocr. Relat. Cancer 10, 179-186 (2003).

29. Finn, R. S., Aleshin, A. \& Slamon, D. J. Targeting the cyclin-dependent kinases (CDK) 4/6 in estrogen receptor-positive breast cancers. Breast Cancer Res. 18, 17 (2016).

30. Sabbah, M., Courilleau, D., Mester, J. \& Redeuilh, G. Estrogen induction of the cyclin D1 promoter: involvement of a cAMP response-like element. Proc. Natl Acad. Sci. USA 96, 11217-11222 (1999).

31. Lundgren, K. et al. Effects of cyclin D1 gene amplification and protein expression on time to recurrence in postmenopausal breast cancer patients treated with anastrozole or tamoxifen: a TransATAC study. Breast Cancer Res. 14, R57 (2012).

32. Nawaz, Z., Lonard, D. M., Dennis, A. P., Smith, C. L. \& O’Malley, B. W. Proteasome-dependent degradation of the human estrogen receptor. Proc. Natl Acad. Sci. USA 96, 1858-1862 (1999).

33. Massarweh, S. et al. A phase II neoadjuvant trial of anastrozole, fulvestrant, and gefitinib in patients with newly diagnosed estrogen receptor positive breast cancer. Breast Cancer Res. Treat. 129, 819-827 (2011).

34. Campbell, R. A. et al. Phosphatidylinositol 3-kinase/AKT-mediated activation of estrogen receptor alpha: a new model for anti-estrogen resistance. J. Biol. Chem. 276, 9817-9824 (2001).

35. Yamnik, R. L. et al. S6 kinase 1 regulates estrogen receptor alpha in control of breast cancer cell proliferation. J. Biol. Chem. 284, 6361-6369 (2009).

36. Zheng, X. Q. et al. Aurora-A is a determinant of tamoxifen sensitivity through phosphorylation of ERalpha in breast cancer. Oncogene 33, 4985-4996 (2014).

37. Gradishar, W. J. et al. NCCN Guidelines Insights Breast Cancer, Version 1.2016. J. Natl. Compr. Canc. Netw. 13, 1475-1485 (2015).

38. Osborne, C. K. \& Schiff, R. Mechanisms of endocrine resistance in breast cancer. Annu. Rev. Med. 62, 233-247 (2011).

39. Meng, S. et al. HER-2 gene amplification can be acquired as breast cancer progresses. Proc. Natl Acad. Sci. USA 101, 9393-9398 (2004).

40. Gutierrez, M. C. et al. Molecular changes in tamoxifen-resistant breast cancer: relationship between estrogen receptor, HER-2, and p38 mitogen-activated protein kinase. J. Clin. Oncol. 23, 2469-2476 (2005).

41. Satyanarayana, A. \& Kaldis, P. Mammalian cell-cycle regulation: several Cdks, numerous cyclins and diverse compensatory mechanisms. Oncogene 28, 2925-2939 (2009).

42. Cancer Genome Atlas, N. Comprehensive molecular portraits of human breast tumours. Nature 490, 61-70 (2012).

43. Raspe, E. et al. CDK4 phosphorylation status and a linked gene expression profile predict sensitivity to palbociclib. EMBO Mol. Med. 9, 1052-1066 (2017).

44. Kato, J. Y., Matsuoka, M., Strom, D. K. \& Sherr, C. J. Regulation of cyclin Ddependent kinase 4 (cdk4) by cdk4-activating kinase. Mol. Cell. Biol. 14, 2713-2721 (1994).

45. Salzano, M. et al. Vaccinia-related kinase 1 (VRK1) confers resistance to DNA-damaging agents in human breast cancer by affecting DNA damage response. Oncotarget 5, 1770-1778 (2014).

46. Fournier, M. V. et al. Gene expression signature in organized and growtharrested mammary acini predicts good outcome in breast cancer. Cancer Res. 66, 7095-7102 (2006).

47. Finetti, P. et al. Sixteen-kinase gene expression identifies luminal breast cancers with poor prognosis. Cancer Res. 68, 767-776 (2008).

48. Liao, Y. \& Hung, M. C. A new role of protein phosphatase 2a in adenoviral E1A protein-mediated sensitization to anticancer drug-induced apoptosis in human breast cancer cells. Cancer Res. 64, 5938-5942 (2004).

49. Yu, Q. et al. Requirement for CDK4 kinase function in breast cancer. Cancer Cell 9, 23-32 (2006).

50. Rudas, M. et al. Cyclin D1 expression in breast cancer patients receiving adjuvant tamoxifen-based therapy. Clin. Cancer Res. 14, 1767-1774 (2008).

51. Stendahl, M. et al. Cyclin D1 overexpression is a negative predictive factor for tamoxifen response in postmenopausal breast cancer patients. Br. J. Cancer 90, 1942-1948 (2004).

52. Hui, R. et al. Constitutive overexpression of cyclin D1 but not cyclin E confers acute resistance to antiestrogens in T-47D breast cancer cells. Cancer Res. 62, 6916-6923 (2002).

53. Holz, M. K. The role of S6K1 in ER-positive breast cancer. Cell Cycle 11, 3159-3165 (2012).
54. Wang, L. H. et al. The mitotic kinase Aurora-A induces mammary cell migration and breast cancer metastasis by activating the Cofilin-F-actin pathway. Cancer Res. 70, 9118-9128 (2010).

55. D'Assoro, A. B. et al. The mitotic kinase Aurora--a promotes distant metastases by inducing epithelial-to-mesenchymal transition in ERalpha(+) breast cancer cells. Oncogene 33, 599-610 (2014).

56. Tifft, K. E., Bradbury, K. A. \& Wilson, K. L. Tyrosine phosphorylation of nuclear-membrane protein emerin by Src, Abl and other kinases. J. Cell Sci. 122, 3780-3790 (2009).

57. Jin, K. et al. HOXB7 is an ERalpha cofactor in the activation of HER2 and multiple ER target genes leading to endocrine resistance. Cancer Discov. 5, 944-959 (2015).

58. Jin, K. et al. The HOXB7 protein renders breast cancer cells resistant to tamoxifen through activation of the EGFR pathway. Proc. Natl Acad. Sci. USA 109, 2736-2741 (2012).

59. $\mathrm{Wu}, \mathrm{X}$. et al. Chromodomain helicase DNA binding protein 5 plays a tumor suppressor role in human breast cancer. Breast Cancer Res. 14, R73 (2012).

60. Su, D. et al. Role of ERRF, a novel ER-related nuclear factor, in the growth control of ER-positive human breast cancer cells. Am. J. Pathol. 180, 1189-1201 (2012).

\section{Acknowledgements}

The authors thank Drs. Yushan Zhu and Jun Zhou for providing plasmids and materials The authors thank Dr Tao Zhu for providing MCF7-TAMR cell model. The study was supported by the National Natural Science Foundation of China (Grant No. 91649107 and 81402320), the Natural Science Foundation of Tianjin City of China (Grant No. 17JCYBJC24100 and 16JCYBJC27000), and the Strategic Priority Research Program (Pilot study) "Biological basis of aging and therapeutic strategies" of the Chinese Academy of Sciences (grant XDPB10).

\section{Author contributions}

Z.Z., J.T.D., and J.H. conceived and designed the study. A.G., T.S., and G.M. performed cell culture experiments, histology, immunohistochemistry, and analysis. A.G. and J.C. performed Mouse xenograft studies. Z.Z. and L.C. contributed tissue array experiments and recording data analysis. Z.Z. and Y.W. performed Kaplan-Meier analysis. A.G. and Q.W. performed cell cycle analysis. Q.H., T.S., Q.W., and J.S. contributed the GSTpulldown and IP and results. A.G. and R.W. contributed CHX chase assay and results. J. Z., L.L., J.T.D. provided comments on the manuscript. Z.Z., J.H., and A.G. contributed manuscript preparation.

\section{Additional information}

Supplementary Information accompanies this paper at https://doi.org/10.1038/s41467018-06309-8.

Competing interests: The authors declare no competing interests.

Reprints and permission information is available online at http://npg.nature.com/ reprintsandpermissions/

Publisher's note: Springer Nature remains neutral with regard to jurisdictional claims in published maps and institutional affiliations.

\footnotetext{
Open Access This article is licensed under a Creative Commons AY Attribution 4.0 International License, which permits use, sharing,
adaptation, distribution and reproduction in any medium or format, as long as you give appropriate credit to the original author(s) and the source, provide a link to the Creative Commons license, and indicate if changes were made. The images or other third party material in this article are included in the article's Creative Commons license, unless indicated otherwise in a credit line to the material. If material is not included in the article's Creative Commons license and your intended use is not permitted by statutory regulation or exceeds the permitted use, you will need to obtain permission directly from the copyright holder. To view a copy of this license, visit http://creativecommons.org/ licenses/by/4.0/.
}

(c) The Author(s) 2018 